\title{
What is the potential for bisexual men in China to act as a bridge of HIV transmission to the female population? Behavioural evidence from a systematic review and meta-analysis
}

\author{
Eric PF Chow ${ }^{1}$, David P Wilson ${ }^{1}$ and Lei Zhang ${ }^{1,2^{*}}$
}

\begin{abstract}
Background: HIV prevalence among men who have sex with men (MSM) in China has rapidly increased in recent years. It is suggested that MSM could be a potential bridge of HIV transmission to the general female population. We investigated the bisexual behaviour of MSM in China through systematic review and meta-analysis.

Methods: We conducted a systematic review and meta-analyses on published peer-reviewed Chinese and English literature during 2001-2010 according to the PRISMA guidelines. Marital status and sexual behavioural indicators of MSM were presented graphically using forest plots. The pooled effect rates with $95 \%$ confidence intervals were also calculated. Meta-regression analyses were performed to examine the factors associated with high heterogeneities across the studies.

Results: Forty-three eligible articles (11 in English and 32 in Chinese) were identified. Our results showed that $17.0 \%$ (95\% Cl: 15.1-19.1\%) of MSM in China are currently married to a woman and 26.3\% (95\% Cl: 23.6-29.1\%) of MSM had female sexual partners in the last six months. The pooled estimates for condom use rate between MSM and female sex partners was $41.4 \%$ (95\% Cl: $35.5-47.5 \%)$ at the last sex act; and $25.6 \%$ (95\% Cl: 23.0-28.4\%) in the last six months. The consistent condom use rates with regular, non-commercial, casual and commercial female sex partners in the last six months were 23.3\% (95\% Cl: 11.25-42.1\%), 39.0\% (95\% Cl: 28.8-50.3\%) and 55.8\% (95\% Cl: 41.4-69.4\%), respectively.

Conclusions: A substantial proportion of Chinese MSM is currently married or had sexual relations with a female in the past six months. In addition, low condom usage was common between married MSM and their wives, hence posing a higher risk of transmitting HIV. Harm-reduction programs targeting married MSM and their female partners are necessary to curb the further spread of HIV infection to the general female population.
\end{abstract}

Keywords: Men who have sex with men (MSM), meta-analysis, bisexual behaviour, condom use

\section{Background}

Although blood transfusions and injecting drug use had historically been the dominant risk factors associated with HIV infection in China, sexual transmission has become the main route of HIV transmission in recent years. Among the 48,000 (41,000-55,000) Chinese people newly diagnosed with HIV in 2009 [1], $42.2 \%$ and $32.5 \%$

\footnotetext{
* Correspondence: Izhang@kirby.unsw.edu.au

'The Kirby Institute for infection and immunity in society, University of New South Wales, Sydney, Australia

Full list of author information is available at the end of the article
}

were due to heterosexual and homosexual transmission respectively [1,2]. Notably, HIV prevalence among men who have sex with men (MSM) in China had increased rapidly from $1.4 \%$ to $5.3 \%$ between 2001-2009 [3] and this increasing trend is likely to continue in coming years.

The rapid increase in HIV prevalence among Chinese MSM was probably due to many factors including the increasing numbers of men who engage in homosexual sex; multiple male partners; and high levels of unprotected anal intercourse. A recent systematic review and

\section{C) Biomed Central}


meta-analysis study showed that just over one-third of MSM report consistent condom use with their male sex partners in the last six months and only $20 \%$ consistently used condoms with their regular male partners [4]. It has also been reported that sexually active Chinese MSM report having an average of six to seven male sexual partners in the last six months and $18 \%$ of them had participated in group sex in the past 12 months [5].

Due to traditional cultural and family values, Chinese MSM often marry to conceal their homosexuality from family and friends. It was estimated that approximately $50-70 \%$ of MSM have had sex with females in their lifetime [6-10] and 70-90\% will eventually marry $[11,12]$. This suggests that bisexual behaviours among Chinese MSM are common. As they are often the only son in their families, as a direct result of China's one-child policy, they also face their parents' expectation to have children to continue the family line.

Chinese MSM tend to have low rates of condom use with their female partners [13]. Given the rapid increase in HIV prevalence among Chinese MSM, their bisexual behaviours could potentially act as a bridge of HIV transmission to the general population $[5,9,14-16]$. In this study we conducted a systematic review of studies on bisexual behaviours among Chinese MSM in both Chinese and English literature to examine the extent to which bisexual behaviour is common among Chinese MSM.

Our review, coupled with meta-analyses for specific questions, summarised evidence of bisexual behaviours of Chinese MSM. These included the age of MSM who are currently married; the percentage of Chinese MSM who had female sexual partners in last six months; the percentage of MSM who are currently married; and the condom usage rate among MSM with their female sexual partners at the last sex act or in the last six months. Different types of partnerships, including regular, non-commercial casual or commercial, were also distinguished in our review and analysis. In comparison with a recent published systematic review on behavioural studies among Chinese MSM [17], this study employed a metaanalysis approach with a strong focus on the condom use with different types of female partners. In addition, this study substantially extended the previous analysis to include both English and Chinese literature. Our study provides more detail insights and quantifiable results on the bisexual behaviours among MSM in China.

\section{Method}

\section{Searching strategy}

Two investigators (EPFC, LZ) performed a systematic literature search for peer-reviewed studies from the following electronic databases: PubMed, Wanfang Data, China National Knowledge Infrastructure (CNKI) and Chinese
Scientific Journals Fulltext Database (CQVIP), published between 2001 and 2010. The keywords searched in the databases were ("unprotected sex" $O R$ "condom use" $O R$ "risk behaviour") OR ("KABP [knowledge, attitudes, beliefs and practices]" OR "behaviour") $O R$ ("Married" OR "Unmarried") $O R$ ("female sexual partners") $A N D$ ("homosexual" OR "MSM" OR "men who have sex with men" OR "gay") $A N D$ "China". The references in the relevant articles were searched manually. We limited our search to articles published in Chinese and English only. We followed the PRISMA (Preferred Reporting Items for Systematic Reviews and Meta-Analyses) guidelines issued in 2009 for conducting and reporting this systematic review and meta-analysis [18].

\section{Study selection}

Studies were included if they (1) reported the percentage of condom use (at the last sex act and/or consistent condom use in the last six months) $O R$ (2) reported the percentage of MSM who had female sexual partners in last six months, through a peer-reviewed publication of cohort or cross-sectional study. We excluded studies in which the target study population was specifically money boys or MSM drug users only. Review papers, non-peer reviewed theses, local reports, conference abstract and presentations were excluded from this study. The two duration types of condom use in "last sex act" and "in last six months" were the most frequent and standardised reported indicators in most Chinese and English literature. Therefore consistent condom use rates within other durations (e.g. past one, three, twelve months or others) were also excluded. Studies which did not report study location, time periods and sample size were also excluded. If the study was duplicated in the databases, studies published in Chinese or published latest were excluded from this review.

\section{Quality assessment}

The quality of studies was assessed using a validated quality assessment tool for cross-sectional studies [19]. The following seven items were assessed to calculate a total quality score: (1) clear definition of the target population; (2) representativity of probability sampling; (3) sample characteristics matching the overall population; (4) adequate response rate; (5) standardised data collection methods; (6) reliable and valid survey measures/instruments; (7) appropriate statistical methods. Answers were scored 0 and 1 for 'No' and 'Yes', respectively. The total quality score varied between 0 and 7 (see Table 1).

\section{Data abstraction}

For all identified studies, we also extracted information about the study: first author and year of publication, 
Table $1 \mathrm{~A}$ summary of studies reporting bisexual behaviour among men who have sex with men in China

\begin{tabular}{|c|c|c|c|c|c|c|c|c|c|c|c|c|}
\hline \multirow[b]{2}{*}{$\begin{array}{l}\text { First } \\
\text { author, } \\
\text { published } \\
\text { year }\end{array}$} & \multicolumn{6}{|l|}{ Study Design } & \multicolumn{2}{|c|}{ Sexual orientation $\%(\mathrm{n} / \mathrm{N})$} & \multicolumn{3}{|c|}{ Condom usage $\%(\mathrm{n} / \mathrm{N})$} & \multirow{2}{*}{$\begin{array}{l}\text { Quality } \\
\text { Score }\end{array}$} \\
\hline & $\begin{array}{l}\text { Study Period } \\
\text { (MM/YY) }\end{array}$ & Study location & $\begin{array}{l}\text { Age } \\
\text { range } \\
\text { (Mean) }\end{array}$ & $\begin{array}{l}\text { Recruitment } \\
\text { method }^{1}\end{array}$ & $\begin{array}{l}\text { Sampling } \\
\text { method }^{2}\end{array}$ & $\begin{array}{l}\text { Sampling } \\
\text { size }\end{array}$ & $\begin{array}{l}\text { Percentage } \\
\text { of married } \\
\text { MSM }\end{array}$ & $\begin{array}{l}\text { Had female } \\
\text { partners in last } \\
\text { six months }\end{array}$ & $\begin{array}{l}\text { Types of } \\
\text { female } \\
\text { partner }^{3}\end{array}$ & $\begin{array}{l}\text { Condom } \\
\text { use } \\
\text { at last } \\
\text { sex act }\end{array}$ & $\begin{array}{l}100 \% \text { condom } \\
\text { use in last six } \\
\text { months }\end{array}$ & \\
\hline \multicolumn{13}{|l|}{ East China } \\
\hline $\begin{array}{l}\text { Cai GF, } \\
2008 \text { [26] }\end{array}$ & 06/2006-12/2006 & Zhejiang & $16-46$ & MSM Venues & & 152 & $16.4 \%(25 / 152)$ & $18.9 \%(28 / 148)$ & Commercial & - & $33.3 \%(2 / 6)$ & 4 \\
\hline $\begin{array}{l}\text { Chen SC, } \\
2007[25]\end{array}$ & 07/2005-09/2005 & Hangzhou & $\begin{array}{l}18-70 \\
(28.0)\end{array}$ & $\begin{array}{l}\text { MSM Venues, } \\
\text { internet } \\
\text { invitation }\end{array}$ & Snowball & 365 & $26.8 \%(97 / 362)$ & $11.5 \%(42 / 365)$ & Commercial & $\begin{array}{l}75.0 \%(3 / \\
4)\end{array}$ & $44.4 \%(4 / 9)$ & 4 \\
\hline $\begin{array}{l}\text { Choi KH, } \\
2007 \text { [39] }\end{array}$ & 09/2004-06/2005 & Shanghai & $\begin{array}{l}18-56 \\
(28.0)\end{array}$ & MSM Venues & Snowball & 477 & $13.0 \%(62 / 477)$ & $25.2 \%(120 / 477)$ & - & - & - & 6 \\
\hline $\begin{array}{l}\text { Fu LJ, } 2007 \\
\text { [23] }\end{array}$ & 02/2006-12/2006 & Zhejiang & $\begin{array}{l}19-41 \\
(26.0)\end{array}$ & - & Snowball & 55 & $18.2 \%(10 / 55)$ & $16.4 \%(9 / 55)$ & - & - & - & 3 \\
\hline $\begin{array}{l}\mathrm{Hu} Q, 2006 \\
{[6]}\end{array}$ & 08/2005 & Jiangxi & $\begin{array}{l}15-60 \\
(25.0)\end{array}$ & $\begin{array}{l}\text { MSM Venues, } \\
\text { internet } \\
\text { advertisement }\end{array}$ & Snowball & 200 & $10.5 \%(21 / 200)$ & - & Overall & $\begin{array}{l}51.1 \% \\
(48 / 94)\end{array}$ & - & 4 \\
\hline $\begin{array}{l}\text { Liao MZ, } \\
2006 \text { [27] } \\
\end{array}$ & 09/2005-11/2005 & $\begin{array}{l}\text { Shandong (Jinan, } \\
\text { Yantai, Weihai) }\end{array}$ & $\begin{array}{l}18-73 \\
(26.0) \\
\end{array}$ & MSM Venues & - & 157 & $12.1 \%(19 / 157)$ & $22.9 \%(36 / 157)$ & Commercial & $\begin{array}{l}78.6 \% \\
(11 / 14) \\
\end{array}$ & $50.0 \%(7 / 14)$ & 3 \\
\hline \multirow[t]{2}{*}{$\begin{array}{l}\text { Ruan S, } \\
2009 \text { [16] }\end{array}$} & 03/2007-07/2007 & Jinan & N/A & Peer referral & RDS & 428 & $16.1 \%(69 / 428)$ & $\begin{array}{l}\text { Total: } 33.6 \% \\
\text { (144/428) } \\
\text { [Married: } 97.1 \% \\
\text { (67/69); } \\
\text { Unmarried: } 21.4 \% \\
\text { (77/359)] }\end{array}$ & - & - & - & 5 \\
\hline & 04/2008-06/2008 & Jinan & N/A & Peer referral & RDS & 500 & $\begin{array}{l}25.4 \%(127 / \\
500)\end{array}$ & $\begin{array}{l}\text { Total: } 32.6 \% \\
\text { (163/500) } \\
\text { [Married: } 78.0 \% \\
\text { (99/127); } \\
\text { Unmarried: } 17.2 \% \\
\text { (64/373)] }\end{array}$ & - & - & - & \\
\hline $\begin{array}{l}\text { Wu J, } 2008 \\
{[52]}\end{array}$ & 10/2006-12/2006 & Shanghai & $\begin{array}{l}18-54 \\
(26.6) \\
\end{array}$ & MSM Venues & - & 203 & $11.8 \%(24 / 203)$ & $43.3 \%(88 / 203)$ & Overall & $\begin{array}{l}47.7 \% \\
(42 / 88) \\
\end{array}$ & $34.1 \%(30 / 88)$ & 4 \\
\hline $\begin{array}{l}\text { Yang HT, } \\
2006 \text { [8] }\end{array}$ & 05/2004-07/2004 & Jiangsu & $\begin{array}{l}18-56 \\
(28.0)\end{array}$ & $\begin{array}{l}\text { MSM Venues, } \\
\text { internet } \\
\text { advertisement }\end{array}$ & - & 222 & $21.6 \%(48 / 222)$ & $45.9 \%(102 / 222)$ & Overall & - & $21.3 \%(23 / 108)$ & 5 \\
\hline $\begin{array}{l}\text { Zhu YW, } \\
2007 \text { [53] }\end{array}$ & 03/2006-07/2006 & Jinan & $\begin{array}{l}17-66 \\
(25.4) \\
\end{array}$ & $C D C$ & - & 400 & $11.0 \%(44 / 400)$ & $20.0 \%(80 / 400)$ & Overall & - & $27.5 \%(22 / 80)$ & 4 \\
\hline \multicolumn{13}{|l|}{$\begin{array}{l}\text { Northeast } \\
\text { China }\end{array}$} \\
\hline $\begin{array}{l}\text { Gu Y, } 2004 \\
{[54]}\end{array}$ & 03/2003-04/2003 & Shenyang & $16-49$ & MSM Venues & & 342 & & $36.3 \%(124 / 342)$ & - & - & - & 3 \\
\hline
\end{tabular}


Table 1 A summary of studies reporting bisexual behaviour among men who have sex with men in China (Continued)

\begin{tabular}{|c|c|c|c|c|c|c|c|c|c|c|c|c|}
\hline $\begin{array}{l}\text { Liu LY, } \\
2008 \text { [55] }\end{array}$ & 2006 & Mudanjiang & $\begin{array}{l}\text { N/A } \\
(29.0)\end{array}$ & MSM Venues & - & 202 & $16.8 \%(34 / 202)$ & - & Overall & $\begin{array}{l}26.3 \% \\
(20 / 76)\end{array}$ & $24.0 \%(12 / 50)$ & 2 \\
\hline $\begin{array}{l}\text { Wang J, } \\
2008 \text { [56] }\end{array}$ & 08/2006-10/2006 & Harbin & N/A & MSM Venues & Convenience & 401 & - & $22.4 \%(90 / 401)$ & Overall & $\begin{array}{l}48.9 \% \\
(44 / 90)\end{array}$ & - & 3 \\
\hline $\begin{array}{l}\text { Wang L, } \\
2006[57]\end{array}$ & 07/2004 & Heilongjiang & $17-66$ & MSM Venues & - & 397 & $12.6 \%(50 / 397)$ & $24.7 \%(98 / 397)$ & - & - & - & 3 \\
\hline \multirow{3}{*}{$\begin{array}{l}\text { Zhang D, } \\
2007 \text { [58] }\end{array}$} & 2002 & Harbin & $18-67$ & MSM Venues & - & 215 & - & $41.9 \%(90 / 215)$ & - & - & - & 5 \\
\hline & 2004 & Harbin & $18-67$ & MSM Venues & - & 397 & - & $24.7 \%(98 / 397)$ & - & - & - & \\
\hline & 2006 & Harbin & $18-67$ & MSM Venues & - & 647 & - & $25.0 \%(162 / 648)$ & - & - & - & \\
\hline $\begin{array}{l}\text { Zhao H, } \\
2009 \text { [59] }\end{array}$ & 01/2008-10/2008 & Harbin & $\begin{array}{l}17-52 \\
(25.0) \\
\end{array}$ & VCT Clinic & - & 89 & $7.9 \%(7 / 89)$ & $14.6 \%(13 / 89)$ & Overall & $\begin{array}{l}53.8 \%(7 / \\
13)\end{array}$ & $30.8 \%(4 / 13)$ & 2 \\
\hline \multicolumn{13}{|l|}{ North China } \\
\hline $\begin{array}{l}\text { Guo W, } \\
2008 \text { [60] }\end{array}$ & 10/2007-11/2007 & Hebei & $18-54$ & $\begin{array}{l}\text { MSM Venues, } \\
\text { internet chat } \\
\text { rooms }\end{array}$ & - & 118 & $17.9 \%(19 / 106)$ & $33.0 \%(35 / 106)$ & - & - & - & 3 \\
\hline $\begin{array}{l}\text { Liu H, } 2001 \\
\text { [61] }\end{array}$ & 08/2000-10/2000 & Beijing & $\begin{array}{l}16 / 72 \\
(30.1)\end{array}$ & MSM Venues & TLS & 84 & $29.8 \%(25 / 84)$ & $27.4 \%(23 / 84)$ & - & - & - & 3 \\
\hline $\begin{array}{l}\text { Liu H, } 2005 \\
{[10]}\end{array}$ & 09/2001-01/2002 & Beijing & $\begin{array}{l}18-69 \\
(27.0)\end{array}$ & MSM Venues & - & 481 & $9.4 \%(45 / 481)$ & $30.1 \%(145 / 481)$ & Overall & - & $27.6 \%(40 / 145)$ & 4 \\
\hline \multirow[t]{3}{*}{$\begin{array}{l}\text { Liu H, } 2007 \\
{[28]}\end{array}$} & 08/2005-12/2008 & Beijing & $\begin{array}{l}18-55 \\
(27.2)\end{array}$ & MSM Venues & - & 416 & $11.5 \%(48 / 416)$ & $31.7 \%(132 / 416)$ & Commercial & - & $75.0 \%(12 / 16)$ & 4 \\
\hline & & & & & & & & & Regular & - & $16.5 \%(16 / 97)$ & \\
\hline & & & & & & & & & $\begin{array}{l}\text { Non- } \\
\text { commercial } \\
\text { casual }\end{array}$ & - & $40.7 \%(22 / 54)$ & \\
\hline $\begin{array}{l}\text { Ma J, } 2007 \\
{[62]}\end{array}$ & 04/2006-10/2006 & Tianjin & $\begin{array}{l}\mathrm{N} / \mathrm{A} \\
(25.2)\end{array}$ & $\begin{array}{l}\text { Internet } \\
\text { advertisement }\end{array}$ & - & 433 & $9.5 \%(41 / 433)$ & $22.9 \%(99 / 433)$ & - & - & - & 5 \\
\hline $\begin{array}{l}\text { Qu L, } 2009 \\
\text { [63] }\end{array}$ & 10/2008-12/2008 & Inner Mongolia & $\begin{array}{l}18-63 \\
(27.0) \\
\end{array}$ & MSM Venues & - & 604 & $\begin{array}{l}21.4 \%(129 / \\
604)\end{array}$ & $19.7 \%(119 / 604)$ & Overall & $\begin{array}{l}28.6 \% \\
(34 / 119) \\
\end{array}$ & $19.3 \%(23 / 119)$ & 2 \\
\hline \multirow[t]{2}{*}{$\begin{array}{l}\text { Ruan Y, } \\
2007 \text { [30] }\end{array}$} & 06/2005-11/2005 & Beijing & $\begin{array}{l}17-54 \\
(26.2)\end{array}$ & $\begin{array}{l}\text { MSM Venues, } \\
\text { peer referring, } \\
\text { internet } \\
\text { advertising }\end{array}$ & $\begin{array}{l}\text { Snowball, } \\
\text { convenience }\end{array}$ & 526 & $6.5 \%(34 / 526)$ & $10.6 \%(56 / 526)$ & Regular & - & $32.6 \%(14 / 43)$ & 5 \\
\hline & & & & & & & & & $\begin{array}{l}\text { Non- } \\
\text { commercial } \\
\text { casual }\end{array}$ & - & $34.8 \%(8 / 23)$ & \\
\hline $\begin{array}{l}\text { Wang } \mathrm{CH}, \\
2007[12]\end{array}$ & 06/2003-10/2006 & Chengde & $15-28$ & MSM Venues & Snowball & 82 & $26.8 \%(22 / 82)$ & $22.0 \%(18 / 82)$ & - & - & - & 1 \\
\hline $\begin{array}{l}\text { Wang XL, } \\
2009[64]\end{array}$ & $11 / 2008-12 / 2008$ & Tangshan & $19-53$ & MSM Venues & - & 114 & $50.0 \%(57 / 114)$ & $\begin{array}{l}50.9 \%(58 / 114) \\
-\end{array}$ & & - & - & 2 \\
\hline
\end{tabular}


Table 1 A summary of studies reporting bisexual behaviour among men who have sex with men in China (Continued)

\begin{tabular}{|c|c|c|c|c|c|c|c|c|c|c|c|c|}
\hline \multicolumn{13}{|c|}{ South Central China } \\
\hline $\begin{array}{l}\text { Cai YM, } \\
2009 \text { [65] }\end{array}$ & $10 / 2007-12 / 2007$ & Shenzhen & $\begin{array}{l}18-45 \\
(27.0)\end{array}$ & MSM Venues & RDS & 351 & $21.9 \%(77 / 351)$ & $28.5 \%(100 / 351)$ & Overall & - & $36.8 \%(35 / 95)$ & 3 \\
\hline $\begin{array}{l}\mathrm{He} Q, 2005 \\
{[66]}\end{array}$ & $04 / 2003-05 / 2003$ & Guangzhou & $18-55$ & $\begin{array}{l}\text { Peer referral, } \\
\text { internet } \\
\text { advertisement }\end{array}$ & Snowball & 121 & - & $21.5 \%(26 / 121)$ & Overall & - & $26.9 \%(7 / 26)$ & 4 \\
\hline $\begin{array}{l}\text { Li N, } 2007 \\
{[40]}\end{array}$ & 2006 & Henan & $\begin{array}{l}17-68 \\
(28.3)\end{array}$ & CDC & Continuous & 187 & $31.6 \%(59 / 187)$ & $36.9 \%(69 / 187)$ & - & - & - & 2 \\
\hline $\begin{array}{l}\text { Liu H, } 2009 \\
\text { [45] }\end{array}$ & 2007 & Shenzhen & $18-45$ & MSM Venues & RDS & 293 & $24.9 \%(73 / 293)$ & $25.9 \%(76 / 293)$ & Overall & - & $31.1 \%(91 / 293)$ & 5 \\
\hline $\begin{array}{l}\text { Xing JM, } \\
2007 \text { [41] }\end{array}$ & $10 / 2005-12 / 2005$ & Hunan & $\begin{array}{l}14-65 \\
(29.8)\end{array}$ & $\begin{array}{l}\text { MSM Venues, } \\
\text { internet } \\
\text { invitation }\end{array}$ & - & 372 & $\begin{array}{l}27.3 \%(101 / \\
370)\end{array}$ & $29.0 \%(108 / 372)$ & Overall & - & $32.7 \%(33 / 101)$ & 3 \\
\hline $\begin{array}{l}\text { Xu YF, } \\
2008 \text { [67] }\end{array}$ & 08/2007-09/2007 & Nanning & $17-38$ & $\begin{array}{l}\text { Internet } \\
\text { advertisement, } \\
\text { peer referral }\end{array}$ & - & 230 & $6.1 \%(14 / 230)$ & $13.9 \%(32 / 230)$ & - & - & - & 2 \\
\hline $\begin{array}{l}\text { Zhong F, } \\
2009 \text { [64] }\end{array}$ & 05/2008-08/2008 & Guangzhou & $\geq 18$ & VCT Clinic & RDS & 379 & $21.9 \%(83 / 379)$ & $28.2 \%(107 / 379)$ & - & - & - & 5 \\
\hline \multicolumn{13}{|c|}{ Northwest China } \\
\hline $\begin{array}{l}\text { Miao ZF, } \\
2009 \text { [68] }\end{array}$ & $04 / 2008-06 / 2008$ & Yinchuan & $\begin{array}{l}18-55 \\
(27.7) \\
\end{array}$ & $\begin{array}{l}\text { MSM Venues, } \\
\text { internet }\end{array}$ & Snowball & 312 & $24.4 \%(76 / 312)$ & $37.5 \%(117 / 312)$ & Overall & $\begin{array}{l}47.0 \% \\
(55 / 117) \\
\end{array}$ & - & 2 \\
\hline $\begin{array}{l}\text { Zhang M, } \\
2009 \text { [24] }\end{array}$ & $05 / 2008-06 / 2008$ & Ürumqi & $\begin{array}{l}18-54 \\
(27.4) \\
\end{array}$ & - & RDS & 231 & $11.3 \%(26 / 231)$ & $16.9 \%(39 / 231)$ & - & - & - & 1 \\
\hline \multicolumn{13}{|c|}{ Southwest China } \\
\hline \multirow[t]{2}{*}{$\begin{array}{l}\text { Feng L, } \\
2009 \text { [13] }\end{array}$} & $07 / 2006-11 / 2006$ & Chongqing & $\geq 18$ & $\begin{array}{l}\text { MSM venues, } \\
\text { community } \\
\text { outreach, peer } \\
\text { recruitment, } \\
\text { web-based } \\
\text { recruitment }\end{array}$ & VBS, CABC & 1000 & $\begin{array}{l}18.2 \%(182 / \\
1000)\end{array}$ & $24.5 \%(245 / 1000)$ & Overall & $\begin{array}{l}36.3 \% \\
(89 / 245)\end{array}$ & $23.7 \%(58 / 245)$ & 5 \\
\hline & $07 / 2007-11 / 2007$ & Chongqing & $\geq 18$ & $\begin{array}{l}\text { MSM venues, } \\
\text { community } \\
\text { outreach, peer } \\
\text { recruitment, } \\
\text { web-based } \\
\text { recruitment }\end{array}$ & VBS, CABC & 1044 & $\begin{array}{l}22.7 \%(237 / \\
1044)\end{array}$ & $25.2 \%(263 / 1044)$ & Overall & $\begin{array}{l}28.5 \% \\
(75 / 263)\end{array}$ & $22.4 \%(59 / 263)$ & \\
\hline $\begin{array}{l}\text { Feng } Y, \\
2010 \text { [69] } \\
\end{array}$ & $03 / 2007-07 / 2007$ & Chengdu & $16-44$ & MSM Venues & Snowball & 538 & $19.2 \%(96 / 500)$ & $26.5 \%(136 / 513)$ & - & - & - & 6 \\
\hline $\begin{array}{l}\text { Lau JT, } \\
2009 \text { [70] }\end{array}$ & 2005 & Kunming & $15-75$ & $\begin{array}{l}\text { MSM Venues, } \\
\text { peer referral, } \\
\text { internet } \\
\text { recruitment }\end{array}$ & $\begin{array}{l}\text { VBS, } \\
\text { snowball }\end{array}$ & 387 & $20.4 \%(79 / 387)$ & $36.4 \%(141 / 387)$ & - & - & - & 5 \\
\hline
\end{tabular}


Table 1 A summary of studies reporting bisexual behaviour among men who have sex with men in China (Continued)

\begin{tabular}{|c|c|c|c|c|c|c|c|c|c|c|c|c|}
\hline & 2006 & Kunming & $15-75$ & $\begin{array}{l}\text { MSM Venues, } \\
\text { peer referral, } \\
\text { internet } \\
\text { recruitment }\end{array}$ & $\begin{array}{l}\text { VBS, } \\
\text { snowball }\end{array}$ & 316 & $11.4 \%(36 / 316)$ & $30.1 \%(95 / 316)$ & - & - & - & \\
\hline \multirow[t]{2}{*}{$\begin{array}{l}\text { Wang Y, } \\
2008 \text { [29] }\end{array}$} & 12/2006-01/2007 & Mianyang & $\begin{array}{l}16-57 \\
(24.8)\end{array}$ & $\begin{array}{l}\text { MSM } \\
\text { Community }\end{array}$ & RDS & 201 & $7.5 \%(15 / 199)$ & $12.9 \%(26 / 201)$ & Overall & $\begin{array}{l}57.7 \% \\
(15 / 26)\end{array}$ & $42.3 \%(11 / 26)$ & 4 \\
\hline & & & & & & & & & Commercial & $\begin{array}{l}87.5 \%(7 / \\
8)\end{array}$ & $62.5 \%(5 / 8)$ & \\
\hline $\begin{array}{l}\text { Xiao Y, } \\
2009 \text { [71] }\end{array}$ & $07 / 2006-09 / 2007$ & Chongqing & $\begin{array}{l}18-68 \\
(27.7)\end{array}$ & $\begin{array}{l}\text { MSM Venues, } \\
\text { internet } \\
\text { recruitment, } \\
\text { community } \\
\text { outreach, peer } \\
\text { referral }\end{array}$ & $\begin{array}{l}\text { VBS, } \\
\text { snowball }\end{array}$ & 1692 & $\begin{array}{l}17.1 \%(289 / \\
1692)\end{array}$ & $21.6 \%(364 / 1689)$ & Overall & $\begin{array}{l}35.8 \% \\
(130 / 363)\end{array}$ & $23.5 \%(85 / 362)$ & 6 \\
\hline $\begin{array}{l}\text { Zhou J, } \\
2008 \text { [72] }\end{array}$ & $09 / 2006-12 / 2006$ & Guiyang & $\begin{array}{l}15-49 \\
(24.0)\end{array}$ & $\begin{array}{l}\text { Peer referral, } \\
\text { internet } \\
\text { recruitment }\end{array}$ & - & 406 & $5.2 \%(21 / 406)$ & $7.4 \%(30 / 406)$ & Overall & $\begin{array}{l}63.3 \% \\
(19 / 30)\end{array}$ & - & 5 \\
\hline \multicolumn{13}{|l|}{ Others } \\
\hline $\begin{array}{l}\text { Xiao Y, } \\
2010 \text { [73] }\end{array}$ & 07/2006-09/2006 & $\begin{array}{l}20 \text { cities from } 7 \\
\text { provinces (Gansu, } \\
\text { Inner Mongolia, } \\
\text { Heilongjiang, Jilin, } \\
\text { Liaoning, Ningxia, } \\
\text { Chongqing) }\end{array}$ & $\begin{array}{l}15-68 \\
(28.4)\end{array}$ & $\begin{array}{l}\text { Internet } \\
\text { advertisement, } \\
\text { community } \\
\text { outreach, peer } \\
\text { referral, MSM } \\
\text { Venues }\end{array}$ & $\begin{array}{l}\text { Snowball, } \\
\text { VBS }\end{array}$ & 4983 & $\begin{array}{l}27.2 \%(1354 / \\
4983)\end{array}$ & $\begin{array}{l}26.0 \%(1298 / \\
4983)\end{array}$ & Overall & - & $\begin{array}{l}18.2 \%(231 / \\
1266)\end{array}$ & 6 \\
\hline $\begin{array}{l}\text { Zhang BC, } \\
2007 \text { [74] }\end{array}$ & 2004 & $\begin{array}{l}6 \text { cities } \\
\text { (Chongqing, } \\
\text { Shenyang, Dalian, } \\
\text { Qingdao, Nanjing, } \\
\text { Xi'an) }\end{array}$ & $\begin{array}{l}15-72 \\
(27.6)\end{array}$ & MSM Venues & Snowball & 1389 & $\begin{array}{l}\text { 17.9\% (249/ } \\
1389)\end{array}$ & $42.4 \%(589 / 1389)$ & Overall & - & $23.6 \%(139 / 589)$ & 5 \\
\hline $\begin{array}{l}\text { Zhang BC, } \\
2008 \text { [5] }\end{array}$ & $2005-2006$ & $\begin{array}{l}9 \text { cities (Harbin, } \\
\text { Shenyang, Xi'an, } \\
\text { Zhengzhou, } \\
\text { Shanghai, Nanjing, } \\
\text { Chongqing, Wuhan, } \\
\text { Chengdu) }\end{array}$ & $\begin{array}{l}13-78 \\
(29.1)\end{array}$ & MSM Venues & Snowball & 2250 & $\begin{array}{l}24.7 \%(555 / \\
2246)\end{array}$ & $\begin{array}{l}\text { Total: } 52.1 \% \\
\text { (548/1051) } \\
\text { [Married: } 74.0 \% \\
\text { (344/465); } \\
\text { Unmarried: } 34.8 \% \\
\text { (204/586)] }\end{array}$ & Overall & - & $\begin{array}{l}\text { Total: } 22.6 \% \\
\text { (190/839) } \\
\text { [Married: } 16.2 \% \\
\text { (65/401); } \\
\text { Unmarried: } \\
\text { 28.5\% (125/ } \\
\text { 438)] }\end{array}$ & 5 \\
\hline
\end{tabular}

MSM Venues: including gay bars, parks, sauna, bathroom, dance clubs; VCT Clinic: HIV voluntary counseling and testing clinic

2 RDS: Respondent-Driven Sampling; TLS: time-location sampling; VBS: Venue-based sampling, CABC: Cruising area-based convenience sampling

${ }^{3}$ Overall partnerships represent the combination of regular, non-commercial casual and commercial female partnerships. 
study period, study location, age of MSM, study base, sampling method, sample size, marital status (percentage of currently married MSM), and types of female sexual partners (regular, non-commercial casual, commercial or overall). We divided the studies into six traditional administrative regions (East China, Northeast China, North China, South Central China, Northwest China and Southwest China). See Table 1 for a description of included studies and extracted data.

\section{Quantitative data synthesis}

The data were analysed in the Comprehensive Meta-Analysis software (V2.0, Biostat, Englewood, New Jersey). For every stratified analysis, we tested the significance of heterogeneity by the Cochran's $Q$ statistics $(p<0.10$ indicates significant heterogeneity) and the level of heterogeneity was assessed by the $I^{2}$ statistic [20]. The value of the $I^{2}$ statistic indicates low (25\%), moderate (50\%) and high (75\%) heterogeneity between studies. Due to the presence of heterogeneity in different stratified analyses, random effect models were used to compute the pooled effect rates (i.e. percentage of married MSM, percentage of MSM who had female sexual partners in the last six months and the condom use rates), 95\% confidence intervals (CI) and relative weights of studies. Results were graphically presented in forest plots. Publication bias was tested by the Begg and Mazumdar rank correlation ( $p<0.05$ on the Kendall's tau indicates significant publication bias) where three or more studies contributed to a stratified analysis [21].

Heterogeneity in meta-analysis is a test of the variation between studies. A high level of heterogeneity $\left(I^{2} \geq\right.$ 75.00, $p<0.10$ ) may be due to the factors of the size of study, sampling methods and study base [20]. We investigated factors related to heterogeneity among studies by using meta-regression [22]. Due to insufficient number of studies, we could only perform meta-regression for the meta-analysis on: (1) MSM marriage rate; (2) MSM who had female sexual partners in the last six months; (3) condom use between MSM and female partners at last act; and (4) consistent condom use between MSM and female partners in the last six months. Potential study characteristics associated with high heterogeneity were examined in a multiple variables model. The multiple variables used in this study were the language of publication (Chinese versus English), size of study (< 200 versus $\geq 200$ ), recruitment method (MSM venues versus non-MSM venues versus not reported), sampling method (not reported method versus other methods versus RDS (respondent driven sampling) or snowball method), and study time period (early 2000s (2000$2004)$ versus late 2000s (2005-2009)). The regression coefficient and $p$ values $(p$ value $<0.10$ indicates that the factor is significantly associated with heterogeneity) for each study characteristic on meta-analysis were calculated by the STATA statistical software package (version 10). Results of meta-regression and subgroup meta-analyses based on the potential study characteristics were summarised in Table 2.

We also performed further analyses on the correlation between age and percentage of married MSM by the Spearman correlation coefficient $(r)$.

\section{Results}

\section{Trial Flow/Flow of included studies}

We identified 707 articles from four electronic databases (103 in PubMed, 133 in CQVIP, 231 in CNKI and 240 in Wanfang); we also identified 22 articles from an internet search and reference lists of published articles. Due to duplication of articles and unrelated topics, we excluded 552 articles after screening the titles. We screened the abstracts of the remaining 177 articles, following which 25 articles were excluded because 20 were non peer-reviewed theses and five were conference presentations or abstracts. The remaining 152 articles were eligible for full-text screening; we further excluded 109 articles (64 did not report bisexual behaviour; 38 reported condom use with female partners in last twelve months; four did not report the study site; two reported condom use in last three months and one reported condom use in the last one month only). Finally, we included 43 articles (11 in English and 32 in Chinese) in a qualitative and quantitative synthesis (Figure 1).

\section{Study characteristics}

The sample size of identified studies ranged from 55 to 4983 (median: 365; IQR: 201-480). Among 43 studies, two did not identify the study base $[23,24]$ and approximately $73 \%(30 / 41)$ of studies recruited MSM participants from MSM venues. Five studies [25-29] reported the condom use rate between MSM and female sex workers. Two studies [28,30] reported the condom use rate between MSM and regular/non-commercial casual female sexual partners. Only two studies $[5,16]$ reported the percentage of married and unmarried MSM who had sex with female in the last six months. The majority of studies recruited MSM participants from MSM venues such as gay bars, sauna and bathrooms (Table 1).

\section{Quantitative data synthesis Marital status}

It was estimated that $17.0 \%$ (95\% CI: $15.1-19.1 \%)$ of MSM in China are currently married (Figure 2). High and significant heterogeneity $\left(I^{2}=93.56, p<0.001\right)$ existed and the publication bias was marginally significant $(p=0.03)$. Meta-regression analysis showed that heterogeneity was explained by the size of study ( $\beta=$ $-0.335, p=0.090)$ and recruitment method $(\beta=-0.203$, $p=0.035$ ) (Table 2). Studies with a small sample size 
Table 2 Result of individual variable meta-regression models for each stratified meta-analysis

\begin{tabular}{|c|c|c|c|c|c|c|c|c|}
\hline \multirow{3}{*}{$\begin{array}{l}\text { Study } \\
\text { Characteristic }\end{array}$} & \multicolumn{8}{|c|}{ Stratified meta-analyses } \\
\hline & \multicolumn{2}{|c|}{ Marital Status of MSM } & \multicolumn{2}{|c|}{$\begin{array}{l}\text { Had female partners } \\
\text { in last six months }\end{array}$} & \multicolumn{2}{|c|}{$\begin{array}{l}\text { Condom use } \\
\text { at last sex act }\end{array}$} & \multicolumn{2}{|c|}{$\begin{array}{l}\text { Consistent condom use } \\
\text { in last six months }\end{array}$} \\
\hline & $\begin{array}{c}\text { Pool estimate } \\
\% \\
(95 \% \mathrm{Cl}), \mathrm{n}\end{array}$ & $\begin{array}{c}\text { Meta- } \\
\text { regression } \\
(\beta, p \text {-value })\end{array}$ & $\begin{array}{c}\text { Pool estimate } \\
\% \\
(95 \% \mathrm{Cl}), \mathrm{n}\end{array}$ & $\begin{array}{c}\text { Meta- } \\
\text { regression } \\
(\beta, p \text {-value })\end{array}$ & $\begin{array}{c}\text { Pool estimate } \\
\% \\
(95 \% \mathrm{Cl}), \mathrm{n}\end{array}$ & $\begin{array}{c}\text { Meta- } \\
\text { regression } \\
\text { ( } \beta, p \text {-value) }\end{array}$ & $\begin{array}{c}\text { Pool estimate } \\
\% \\
(95 \% \mathrm{Cl}), \mathrm{n}\end{array}$ & $\begin{array}{c}\text { Meta- } \\
\text { regression } \\
\text { ( } \beta, p \text {-value) }\end{array}$ \\
\hline \multicolumn{9}{|l|}{$\begin{array}{l}\text { Language of } \\
\text { article: }\end{array}$} \\
\hline Chinese & $\begin{array}{c}16.4(13.9-19.3) \\
n=29\end{array}$ & 0.187 & $\begin{array}{c}25.8(21.7-30.4) \\
n=30\end{array}$ & 0.058 & $\begin{array}{c}45.4(37.5-53.6) \\
n=9\end{array}$ & -0.282 & $\begin{array}{c}26.9(23.8-30.2) \\
n=13\end{array}$ & 0.009 \\
\hline English & $\begin{array}{c}18.2(15.2-21.5) \\
n=13\end{array}$ & $p=0.312$ & $\begin{array}{c}26.8(24.2-29.5) \\
n=16\end{array}$ & $p=0.686$ & $\begin{array}{c}33.6(29.0-38.6) \\
n=3\end{array}$ & $p=0.058$ & $\begin{array}{c}23.4(19.1-28.4) \\
n=5\end{array}$ & $p=0.960$ \\
\hline \multicolumn{9}{|l|}{ Sample size: } \\
\hline$<200$ & $\begin{array}{c}19.8(13.1-28.9) \\
n=10\end{array}$ & -0.335 & $\begin{array}{c}25.9(19.8-33.2) \\
n=10\end{array}$ & -0.056 & $\begin{array}{c}45.4(37.5-53.6) \\
n=9\end{array}$ & & $\begin{array}{c}28.6(24.7-32.8) \\
n=11\end{array}$ & -0.313 \\
\hline$\geq 200$ & $\begin{array}{c}16.3(14.3-18.4) \\
n=32\end{array}$ & $p=0.090$ & $\begin{array}{c}26.3(23.4-29.5) \\
n=36\end{array}$ & $p=0.730$ & $\begin{array}{c}33.6(29.0-38.6) \\
n=3\end{array}$ & - & $\begin{array}{c}23.2(20.4-26.3), \\
n=7\end{array}$ & $p=0.139$ \\
\hline \multicolumn{9}{|l|}{$\begin{array}{l}\text { Recruitment } \\
\text { method: }\end{array}$} \\
\hline $\begin{array}{l}\text { MSM } \\
\text { venues }\end{array}$ & $\begin{array}{c}18.7 \begin{array}{c}(16.6-21.0) \\
n=30\end{array}\end{array}$ & & $\begin{array}{c}28.6(25.5-32.0) \\
n=33\end{array}$ & & $\begin{array}{c}38.4(32.8-44.3) \\
n=9\end{array}$ & & $\begin{array}{c}25.1(22.5-27.9), \\
n=15\end{array}$ & \\
\hline $\begin{array}{l}\text { Non MSM } \\
\text { venues }\end{array}$ & $\begin{array}{c}12.6(8.6-18.1), n \\
=10\end{array}$ & -0.203 & $\begin{array}{c}21.1(16.2-26.9), \\
n=11\end{array}$ & -0.139 & $\begin{array}{c}59.4(47.4-70.3) \\
n=3\end{array}$ & 0.368 & $\begin{array}{c}34.2(23.6-46.6) \\
n=3\end{array}$ & 0.107 \\
\hline $\begin{array}{l}\text { Not } \\
\text { reported }\end{array}$ & $\begin{array}{c}13.5(8.4-21.0), n \\
=2\end{array}$ & $p=0.035$ & $\begin{array}{c}16.8(12.9-21.6) \\
n=2\end{array}$ & $p=0.059$ & - & $p=0.068$ & - & $p=0.679$ \\
\hline \multicolumn{9}{|l|}{$\begin{array}{l}\text { Sampling } \\
\text { method: }\end{array}$} \\
\hline $\begin{array}{l}\text { RDS/ } \\
\text { snowball }\end{array}$ & $\begin{array}{c}17.2(14.5-20.2) \\
n=17\end{array}$ & & $\begin{array}{c}25.7(21.7-30.3), \\
n=21\end{array}$ & & $\begin{array}{c}45.9(36.4-55.7) \\
n=4\end{array}$ & & $\begin{array}{c}25.8(21.8-30.4) \\
n=8\end{array}$ & \\
\hline $\begin{array}{l}\text { Other } \\
\text { methods }\end{array}$ & $\begin{array}{c}21.7 \begin{array}{l}(18.1-25.8) \\
n=8\end{array}\end{array}$ & 0.013 & $\begin{array}{c}26.5(22.9-30.3) \\
n=5\end{array}$ & 0.009 & $\begin{array}{c}37.0(27.4-47.7) \\
n=3\end{array}$ & 0.122 & 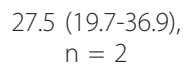 & 0.089 \\
\hline $\begin{array}{l}\text { Not } \\
\text { reported }\end{array}$ & $\begin{array}{c}14.6(11.1-19.0) \\
n=17\end{array}$ & $p=0.886$ & $\begin{array}{c}26.6(22.5-31.1) \\
n=20\end{array}$ & $p=0.899$ & $\begin{array}{c}41.6(28.6-55.9), \\
n=5\end{array}$ & $p=0.128$ & $\begin{array}{c}24.9(21.8-28.4), \\
n=8\end{array}$ & $p=0.268$ \\
\hline \multicolumn{9}{|l|}{ Time period: } \\
\hline $2000-2004$ & $\begin{array}{c}16.8(12.2-22.9) \\
n=5\end{array}$ & 0.023 & $\begin{array}{c}32.6(27.0-38.7) \\
n=9\end{array}$ & -0.236 & - & - & $\begin{array}{c}24.1(21.4-27.1) \\
n=4\end{array}$ & 0.093 \\
\hline 2005-2009 & $\begin{array}{c}17.1(15.0-19.3) \\
n=37\end{array}$ & $p=0.922$ & $\begin{array}{c}24.9(22.1-27.9) \\
n=37\end{array}$ & $p=0.134$ & $\begin{array}{c}41.4(35.5-47.5) \\
n=12\end{array}$ & & $\begin{array}{c}26.1(22.8-29.7) \\
n=14\end{array}$ & $p=0.535$ \\
\hline
\end{tabular}

Table showing the pool estimate (\%), 95\% confidence interval (Cl), number of studies (n), meta-regression coefficient ( $\beta$ ) and significance of $\beta$ ( $p$-value). $p$-values in bold print represent significant associations $(p<0.10)$.

$(<200)$ and participants recruited from MSM venues were more likely to report a higher marriage rate than large studies conducted in non-MSM venues. In addition, a significant association $(r=0.816, p<0.0001)$ between the mean age of the sampled MSM cohorts and the current marital status (Figure 3) was observed, which indicates that older MSM are more likely to be married compared to younger MSM.

\section{Bisexual behaviour of MSM}

The random effects model revealed that approximately 26.3\% (95\% CI: 23.6-29.1\%) of MSM had female sexual partners in the last six months (Figure 4a); no significant publication bias existed $(p=0.353)$. High and significant heterogeneity between studies existed $\left(I^{2}=\right.$
95.27, $p<0.001$ ) in the meta-analysis. Only one study factor (recruitment method) was significantly associated with this heterogeneity $(\beta=-0.139, p=0.059)$ (Table 2). Studies which recruited participants from gay venues were more likely to have female sexual partners in the last six months than MSM recruited from non-gay venues.

A comparison of the bisexual behaviours between married and unmarried MSM showed that married MSM (83.9\%, 95\% CI: 71.1-91.8\%) were more likely to have female sexual partners than unmarried MSM (23.9\%, 95\% CI: $14.7-36.5 \%)$ in last six months (Figure 4b) $\left(\chi^{2}=490.4, p<0.001\right)$. No significant publication bias was observed $(p=0.851)$. 


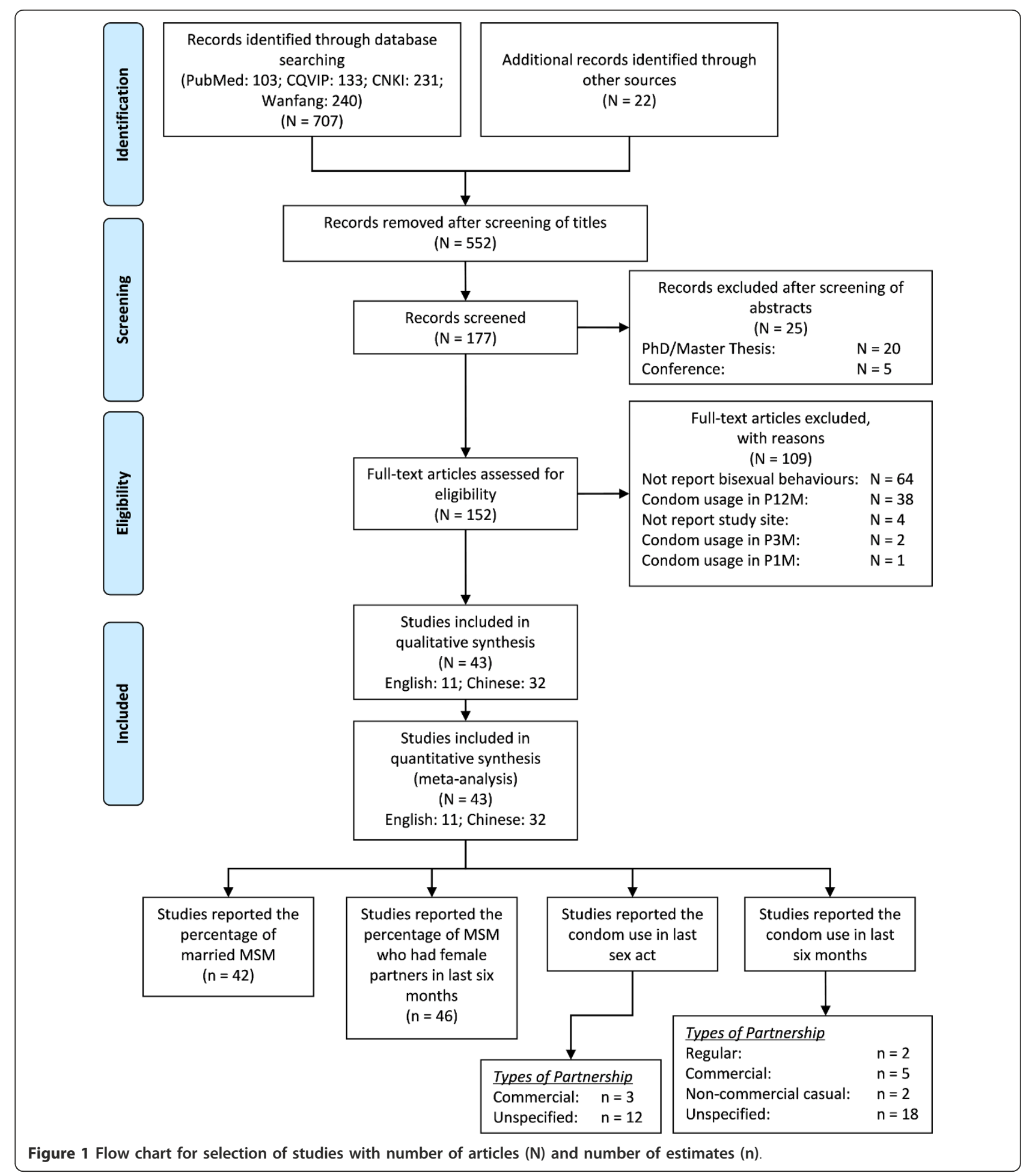

\section{Condom use with female partners}

The pooled estimate of levels of condom use between MSM and their female partners during 2005-2008 was 41.4\% (95\% CI: $35.5-47.5 \%)$ at last sex act (Figure 5a) with significant heterogeneity $\left(I^{2}=78.46, p<0.001\right)$ throughout these studies. Meta-regression analysis showed that two study characteristics, language of articles $(\beta=-0.282, p=0.058)$ and recruitment method $(\beta$ $=0.368, p=0.068)$, significantly contributed to this relatively high heterogeneity (Table 2 ). Since the sample size and time period were collinear with other study factors in the meta-regression model, these two study 


\begin{tabular}{|c|c|c|c|c|c|c|c|c|c|c|c|}
\hline \multirow{2}{*}{\multicolumn{2}{|c|}{ Study name }} & \multirow{2}{*}{\multicolumn{2}{|c|}{ Study period Location }} & \multicolumn{4}{|c|}{ Statistical results } & \multicolumn{3}{|c|}{ Marriage rate and $95 \% \mathrm{Cl}$} & \multirow[b]{2}{*}{$\begin{array}{r}\text { Relative } \\
\text { weight }\end{array}$} \\
\hline & & & & $\begin{array}{c}\text { Event } \\
\text { rate }\end{array}$ & $\begin{array}{c}\text { Lower } \\
\text { limit }\end{array}$ & $\begin{array}{c}\text { Upper } \\
\text { limit }\end{array}$ & $\mathrm{n} / \mathrm{N}$ & & & & \\
\hline & Cai GF, 2008 & 2006 & Zhejiang & 0.164 & 0.114 & 0.232 & $25 / 152$ & $i+$ & $i$ & i & 2.19 \\
\hline & Cai YM, 2009 & 2007 & Shenzhen & 0.219 & 0.179 & 0.266 & $77 / 351$ & $1+$ & i & i & 2.54 \\
\hline & Chen SC, 2007 & 2005 & Hangzhou & 0.268 & 0.225 & 0.316 & $97 / 362$ & + & i & i & 2.58 \\
\hline & Choi KH, 2007 & 2005 & Shanghai & 0.130 & 0.103 & 0.163 & $62 / 477$ & $1+$ & 1 & 1 & 2.52 \\
\hline & Feng L, 2009 & 2006 & Chongqing & 0.182 & 0.159 & 0.207 & $182 / 1000$ & $1+$ & i & i & 2.68 \\
\hline & Feng L, 2009 & 2007 & Chongqing & 0.227 & 0.203 & 0.2532 & $237 / 1044$ & $1+$ & i & i & 2.69 \\
\hline & Feng Y, 2010 & 2007 & Chengdu & 0.192 & 0.160 & 0.229 & $96 / 500$ & $1+$ & i & i & 2.59 \\
\hline & Fu LJ, 2007 & 2006 & Zhejiang & 0.182 & 0.101 & 0.306 & $10 / 55$ & $1+$ & 1 & i & 1.67 \\
\hline & Guo W, 2008 & 2007 & Hebei & 0.179 & 0.117 & 0.264 & $19 / 106$ & $1+$ & i & i & 2.06 \\
\hline & $\mathrm{Hu} Q, 2006$ & 2005 & Jiangxi & 0.105 & 0.069 & 0.156 & $21 / 200$ & $i+$ & i & i & 2.15 \\
\hline & Lau JT, 2009 & 2005 & Kunming & 0.204 & 0.167 & 0.247 & $79 / 387$ & $1+$ & 1 & i & 2.55 \\
\hline & Lau JT, 2009 & 2006 & Kunming & 0.114 & 0.083 & 0.154 & $36 / 316$ & $1+$ & I & i & 2.37 \\
\hline & Li N, 2007 & 2006 & Henan & 0.316 & 0.253 & 0.386 & $59 / 187$ & + & $-i$ & $i$ & 2.44 \\
\hline & Liao MZ, 2006 & 2005 & Shandong & 0.121 & 0.079 & 0.182 & $19 / 157$ & $1+$ & I & 1 & 2.09 \\
\hline & Liu H, 2001 & 2000 & Beijing & 0.298 & 0.210 & 0.403 & $25 / 84$ & $1+$ & -1 & i & 2.12 \\
\hline & Liu H, 2005 & 2002 & Beijing & 0.094 & 0.071 & 0.123 & $45 / 481$ & $i+$ & i & i & 2.45 \\
\hline & Liu H, 2007 & 2008 & Beijing & 0.115 & 0.088 & 0.150 & $48 / 416$ & $1+$ & i & 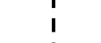 & 2.46 \\
\hline & Liu H, 2009 & 2007 & Shenzhen & 0.249 & 0.203 & 0.302 & $73 / 293$ & $1+$ & I & I & 2.52 \\
\hline & Liu LY, 2008 & 2006 & Mudanjiang & 0.168 & 0.123 & 0.226 & $34 / 202$ & $1+$ & i & i & 2.33 \\
\hline & Ma J, 2007 & 2006 & Tianjin & 0.095 & 0.070 & 0.126 & $41 / 433$ & $1+$ & 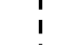 & 1 & 2.42 \\
\hline & Miao ZF, 2009 & 2008 & Yinchuan & 0.244 & 0.199 & 0.294 & $76 / 312$ & $1+$ & i & I & 2.53 \\
\hline & Qu L, 2009 & 2008 & Inner Mongolia & 0.214 & 0.183 & 0.248 & $129 / 604$ & $1+$ & i & i & 2.63 \\
\hline & Ruan S, 2009 & 2007 & Jinan & 0.161 & 0.129 & 0.199 & $69 / 428$ & + & 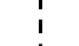 & i & 2.54 \\
\hline & Ruan S, 2009 & 2008 & Jinan & 0.254 & 0.218 & 0.294 & $127 / 500$ & $1+$ & i & i & 2.62 \\
\hline & Ruan Y, 2007 & 2005 & Beijing & 0.065 & 0.047 & 0.089 & $34 / 526$ & it & $i$ & $i$ & 2.37 \\
\hline & Wang $\mathrm{CH}, 2007$ & 2006 & Chengde & 0.268 & 0.184 & 0.374 & $22 / 82$ & $1+$ & -1 & 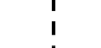 & 2.07 \\
\hline & Wang L, 2006 & 2004 & Heilongjiang & 0.126 & 0.097 & 0.162 & $50 / 397$ & $1+$ & I & I & 2.47 \\
\hline & Wang XL, 2009 & 2008 & Tangshan & 0.500 & 0.409 & 0.591 & $57 / 114$ & i & + & i & 2.33 \\
\hline & Wang Y, 2008 & $2006-2007$ & Mianyang & 0.075 & 0.046 & 0.121 & $15 / 199$ & $1+$ & I & i & 1.99 \\
\hline & Wu J, 2008 & 2006 & Shanghai & 0.118 & 0.081 & 0.170 & $24 / 203$ & $1+$ & i & i & 2.21 \\
\hline & Xiao Y, 2009 & 2007 & Chongqing & 0.171 & 0.154 & 0.1892 & $289 / 1692$ & $i$ & $i$ & i & 2.71 \\
\hline & Xiao Y, 2010 & 2006 & 20 cities & 0.272 & 0.260 & 0.2841 & 1354 / 4983 & 31 & $\begin{array}{l}1 \\
1\end{array}$ & i & 2.76 \\
\hline & Xing JM, 2007 & 2005 & Hunan & 0.273 & 0.230 & 0.321 & $101 / 370$ & + & i & i & 2.58 \\
\hline & Xu YF, 2008 & 2007 & Nanning & 0.061 & 0.036 & 0.100 & $14 / 230$ & it & i & i & 1.96 \\
\hline & Yang HT, 2006 & 2004 & Jiangsu & 0.216 & 0.167 & 0.275 & $48 / 222$ & $1+$ & 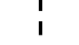 & i & 2.42 \\
\hline & Zhang BC, 2007 & 2004 & 6 cities & 0.179 & 0.160 & 0.2002 & $249 / 1389$ & $1+$ & $\begin{array}{l}1 \\
1\end{array}$ & i & 2.70 \\
\hline & Zhang BC, 2008 & $2005-2006$ & 9 cities & 0.247 & 0.230 & 0.2655 & $555 / 2246$ & $t$ & i & i & 2.74 \\
\hline & Zhang M, 2009 & 2008 & Ürumqi & 0.113 & 0.078 & 0.160 & $26 / 231$ & + & 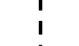 & 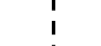 & 2.24 \\
\hline & Zhao H, 2009 & 2008 & Harbin & 0.079 & 0.038 & 0.156 & $7 / 89$ & $1+$ & i & i & 1.50 \\
\hline & Zhong F, 2009 & 2008 & Guangzhou & 0.219 & 0.180 & 0.263 & $83 / 379$ & $1+$ & i & i & 2.56 \\
\hline & Zhou J, 2008 & 2006 & Guiyang & 0.052 & 0.034 & 0.078 & $21 / 406$ & it & 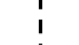 & 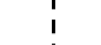 & 2.18 \\
\hline & Zhu YW, 2007 & 2006 & Jinan & 0.110 & 0.083 & 0.145 & $44 / 400$ & $1+$ & i & i & 2.44 \\
\hline & Total $(R=93.56$ & $p<0.001)$ & & 0.170 & 0.151 & 0.191 & & $i$ & $i$ & $i$ & \\
\hline & Test for publicat & tion bias $(p=$ & & & & & & $0 \%$ & $50 \%$ & $100 \%$ & \\
\hline
\end{tabular}

characteristics were both excluded by the meta-regression model. There was no significant publication bias in this meta-analysis $(p=0.273)$.

Consistent condom use with female sexual partners in last six months was $25.6 \%$ (95\% CI: 23.0-28.4\%) during the period of 2002-2008 (Figure 5b). Moderate heterogeneity was observed between studies $\left(I^{2}=70.14, p<0.001\right)$, but we found that none of the study characteristics was significantly associated with this heterogeneity (Table 2 ). There was no significant publication bias observed $(p=0.140)$. 


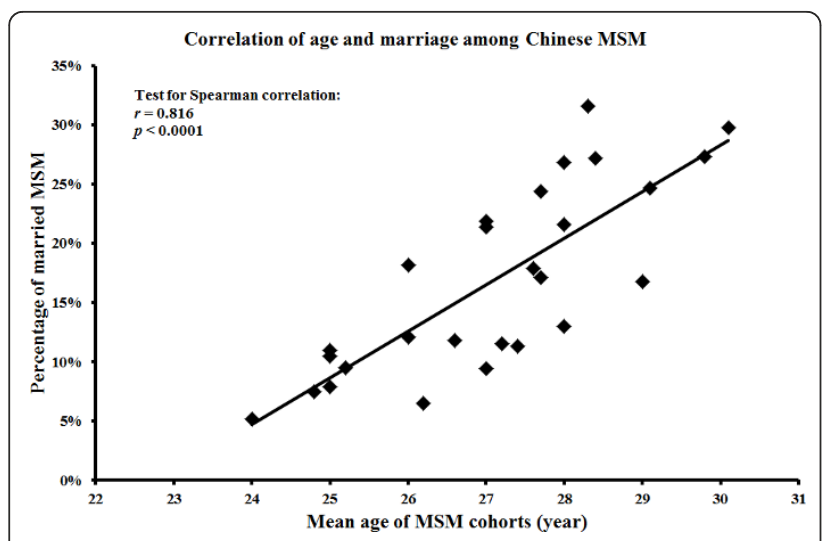

Figure 3 Correlation between mean age of MSM in China and the percentage of MSM who are currently married.

Condom use among MSM with commercial female partners was also analysed according to identified studies (three reported condom use at last act $[25,27,29]$, and five reported rates of consistent use in the last six months [25-29]). The pooled estimates of condom use in commercial partnerships were $80.3 \%$ (95\% CI: 60.491.6\%) $\left(I^{2}=0.00, p=0.602\right)$ at last sex act and $55.8 \%$ (95\% CI: 41.4-69.4\%) $\left(I^{2}=4.50, p=0.327\right)$ in the last six months (Figure 6). No significant publication bias was observed in either of the stratified analyses $(p=0.602$ and 0.327 for last sex act and last six months, respectively). Since heterogeneities were low and not significant in both stratified meta-analyses, meta-regression analysis was not performed.

Two studies reported the consistent condom use with regular and non-commercial causal female partners in the last six months $[28,30]$. In comparison with commercial partnerships, condom usage rates in regular and non-commercial casual partnerships were much lower, estimated to be $23.3 \%$ (95\% CI: $11.25-42.1 \%)\left(I^{2}=77.37, p=0.036\right)$ and 39.0\% (95\% CI: $28.8-50.3 \%)\left(I^{2}=0.00, p=0.240\right)$, respectively (Figure 7). Publication bias could not be assessed due to lack of studies in both stratified meta-analyses.

\section{Discussion}

Our meta-analysis showed that approximately $17.0 \%$ of MSM are currently married to a female in China, which is consistent with another recent review which reported an average of $17.5 \%$ [17]. In contrast, the percentage of MSM who are married in most Western developed countries is much lower. Only $1.5-3.0 \%$ of MSM are currently married to a woman in the United States [31-33] and 7.9\% in Australia [34]; however, higher rates of marriage among MSM were observed in some other developing countries (for example, $35-42 \%$ in India $[35,36])$. There is generally greater acceptance and legitimacy of gay identity, gay marriage or cohabitation relationships and less social stigma, discrimination and family pressure in developed countries. Previous studies showed that only $11-17 \%$ of Chinese MSM disclosed their homosexual sexual orientation to their wives $[37,38]$ and $1 \%$ of them disclosed to their children [37]. Due to the high prevalence of bisexual behaviours among MSM in China, this group is very likely to facilitate HIV transmission to the female population to a much greater extent than occurs in most countries where HIV predominantly affects MSM. Our review provides a timely synthesis of current evidence of bisexual behaviours among MSM in China, which could inform the implementation of effective intervention programs.

Previous available studies indicated that Chinese MSM had an average of 1-5 female sexual partners in the last six months $[7,8,39,40]$. Additionally, we estimated that a higher proportion (83.9\%) of married MSM have had female sexual partners than unmarried MSM (23.9\%). However, only $16.2 \%$ and $28.5 \%$ of married and unmarried MSM have had consistent condom use with their female partners in the past six months, respectively [5]. Fifty-eight percent of married MSM had concurrent male and female sexual partners in last six months, hence putting their wives at a greater risk of HIV infection [41].

Partnership type had strong influences on condom use of MSM. In general, condom usage at last sex act between MSM and overall female sexual partners was estimated to be $41.4 \%$ (95\% CI: $35.5-47.5 \%$ ); but only 25.6\% (95\% CI: 23.0-28.4\%) of MSM used condoms consistently in the last six months. A study in 2004 found that $31.8 \%$ of MSM had regular female sex partners, $6.0 \%$ had non-commercial casual female sex partners and $4.5 \%$ had commercial female sex partners [14]. Among three specific partnership types, consistent condom use was estimated to be the greatest in commercial partnerships (55.8\%, 95\% CI: 41.4-69.4\%), followed by non-commercial casual partnerships $(39.0 \%$, 95\% CI: $28.8-50.3 \%)$ and regular partnerships $(23.3 \%, 95 \% \mathrm{CI}$ : 11.2-42.1\%). In general, reasons of low condom use with partners mainly included: emotional and physical sensation, availability of condoms, and loyalty and trust $[5,25]$. In addition, sexual abuse was common in China. It was reported that $30 \%$ of wives were abused by their homosexual or bisexual husbands to relieve their mental pressure from social stigma and discrimination [42]. This indicated that regular sexual partners of Chinese MSM were often not empowered to negotiate for protected sexual intercourse with their male partners.

Several limitations of our study must be noted. First, the selected studies in this review recruited MSM participants predominantly from major urban Chinese cities. Bisexual behaviour such as MSM who have female 


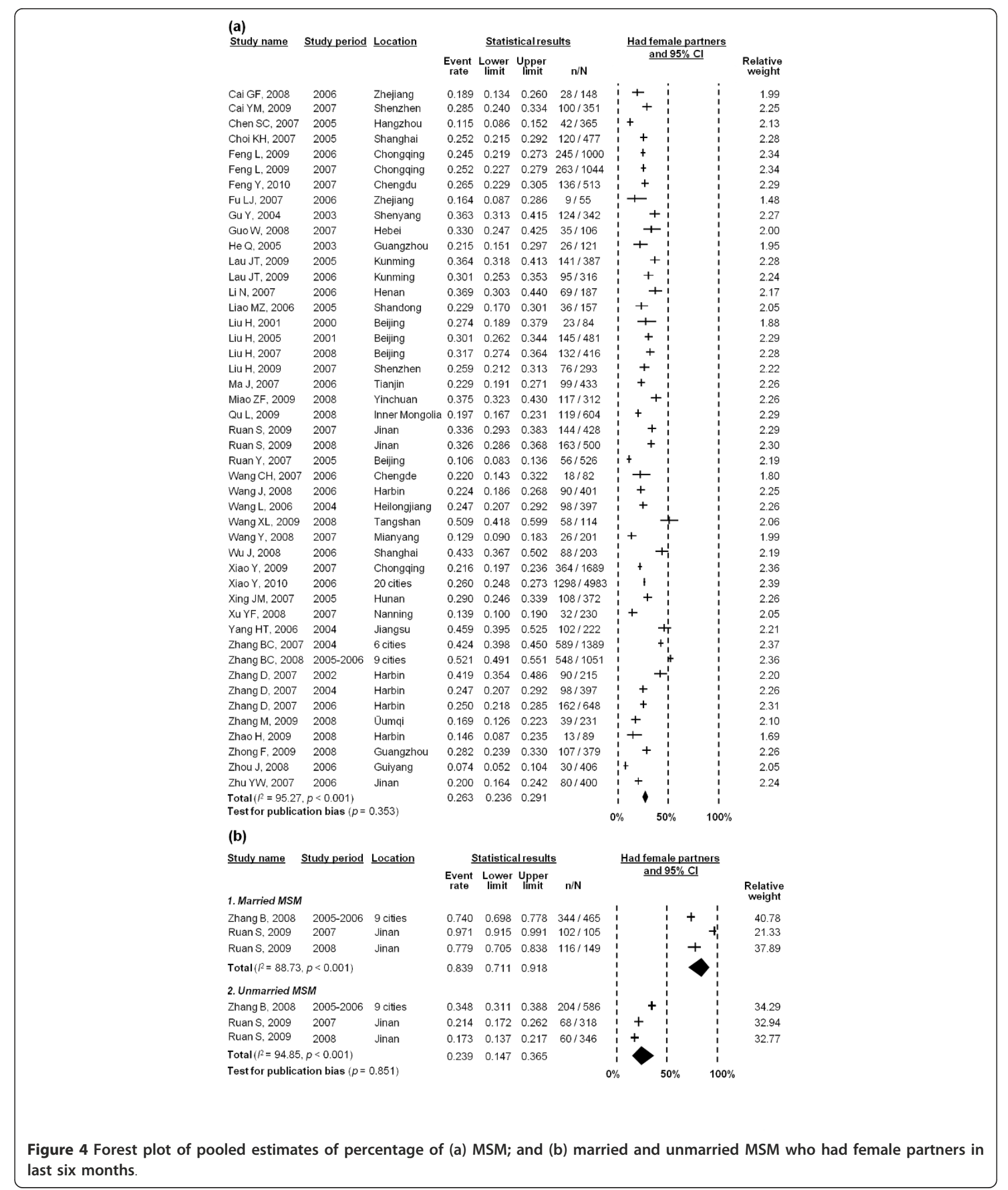

sexual partners and condom usage may be significantly different between urban and rural regions. Second, based on our search criteria, we could not identify any studies on condom use at the last sex act in the South
Central region or consistent condom use in the last six months in the Northwest region. Our pooled estimates of condom usage may not be representative for the entire MSM population in China. Third, very few 


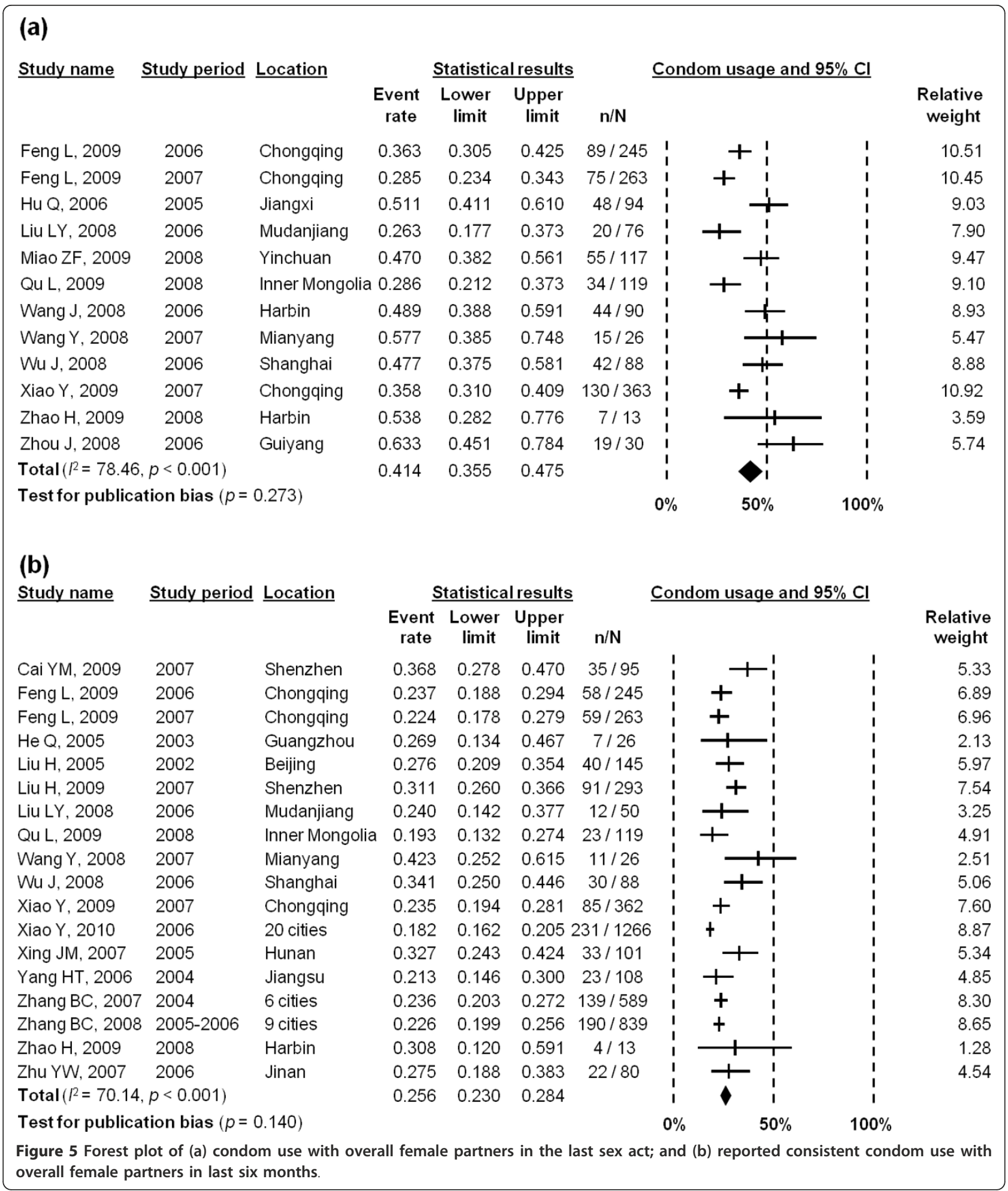

studies reported bisexual behaviours stratified according to their marital status and partnership types. This substantially limited the strength of meta-analysis in these cases. Fourth, due to insufficient description of some studies, we were unable to classify whether the sexual contacts between MSM and female was penile-vaginal intercourse, penile-anal intercourse or oral sex. Male-tofemale anal contacts often had a higher risk of HIV 


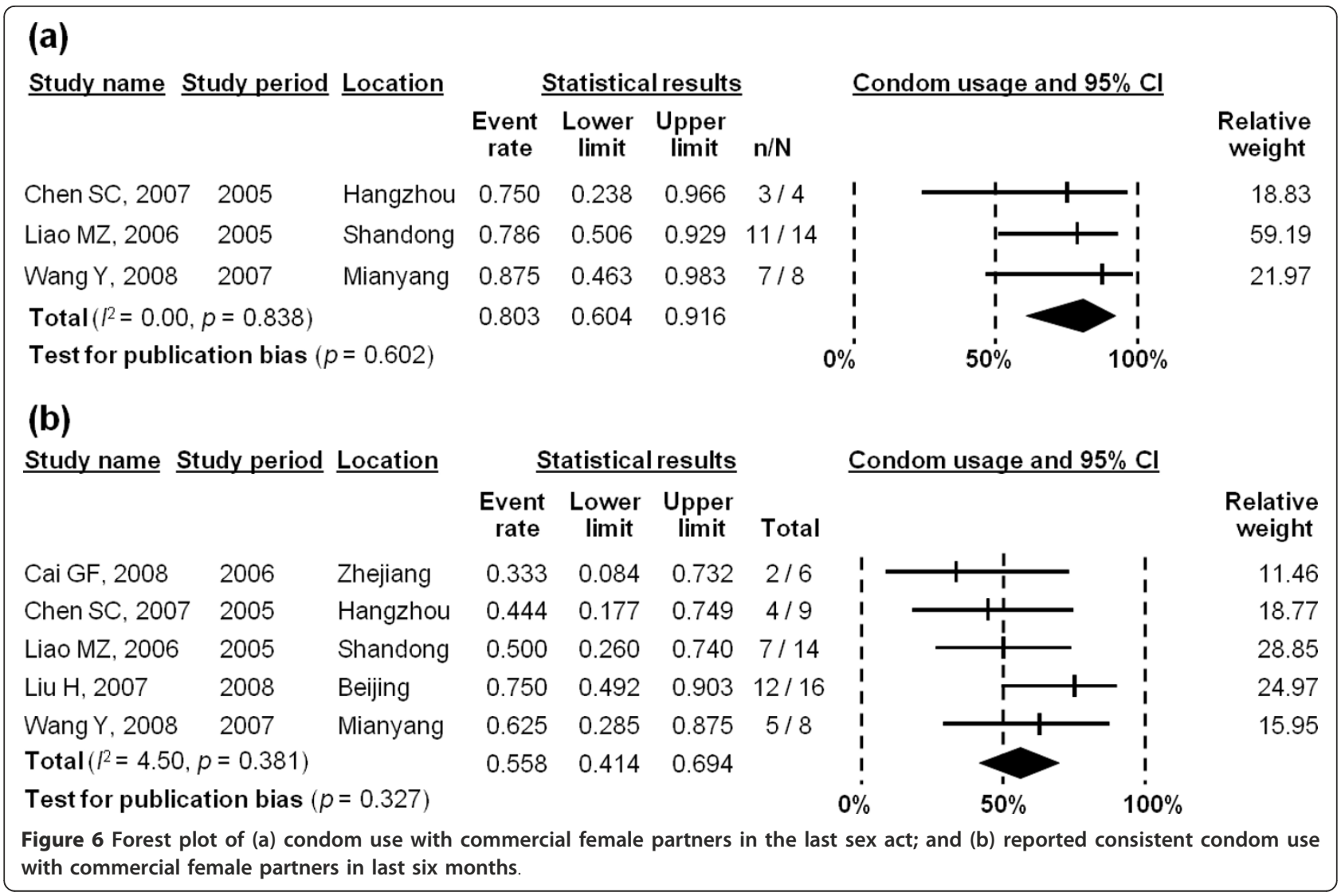

\section{(a)}

Study name Study period Location

$\begin{array}{llllccc} & & & \begin{array}{c}\text { Event } \\ \text { rate }\end{array} & \begin{array}{c}\text { Lower } \\ \text { limit }\end{array} & \begin{array}{c}\text { Upper } \\ \text { limit }\end{array} & \mathbf{n} / \mathbf{N} \\ \text { Liu } \mathrm{H}, 2007 & 2008 & \text { Beijing } & 0.165 & 0.104 & 0.252 & 16 / 97 \\ \text { Ruan } Y, 2007 & 2005 & \text { Beijing } & 0.326 & 0.203 & 0.477 & 14 / 43 \\ \operatorname{Total}\left(P^{2}=77.37, p=0.036\right) & & 0.233 & 0.112 & 0.421 & \end{array}$

(b)

Study name Study period Location

$\begin{array}{cccc}\begin{array}{c}\text { Event } \\ \text { rate }\end{array} & \begin{array}{c}\text { Lower } \\ \text { limit }\end{array} & \begin{array}{c}\text { Upper } \\ \text { limit }\end{array} & \mathbf{n} / \mathbf{N} \\ 0.407 & 0.285 & 0.542 & 22 / 54 \\ 0.348 & 0.184 & 0.557 & 8 / 23 \\ 0.390 & 0.288 & 0.503 & \end{array}$

\section{Condom usage and $95 \% \mathrm{Cl}$}

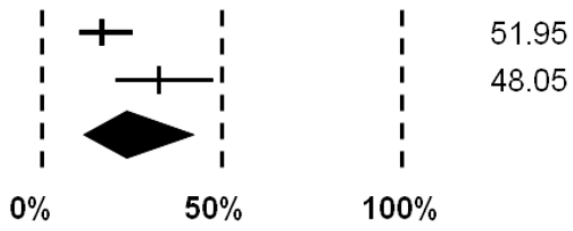

\section{Condom usage and $95 \% \mathrm{Cl}$}

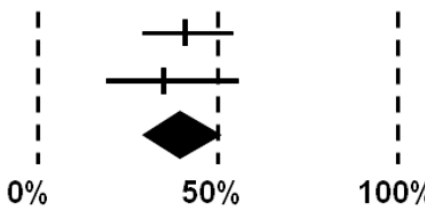

Relative weight

71.42 28.58

Figure 7 Forest plot of condom use with (a) regular female partners; and (b) non-commercial casual female partners in last six months. 
transmission than vaginal contact [43]. Due to reproductive reasons, MSM might not use condoms during vaginal sex but they may use condoms in anal sex. Since vaginal intercourse was more common than anal intercourse in male-female partnership, we assumed all condom usage estimates were associated with vaginal sexual intercourse. Fifth, due to limited number of studies available, we were unable to investigate the associations between the age of MSM and consistent condom use in different geographical locations and with different types of partnerships. For the same reason, the contribution of age to the heterogeneity of the studies could not be investigated. Sixth, our study only addressed the overall MSM population; specific populations such as male sex workers ('money boys') were not included in this study. In China, 'money boys' have become one of the emerging high-risk sub-populations in gay communities in recent years [44]. However, very few epidemiological and socio-behavioural studies have focused on this population to date. Limited studies indicated that Chinese 'money boys' are usually younger than the overall MSM population and unmarried [38,45-47]. An estimated $71 \%$ of Chinese money boys had 1-3 male clients and $11 \%$ had more than 10 clients per week [38]. Approximately $27 \%$ of money boys had female clients in last six months [48]. Unprotected sex with both male and female clients was very common $(59.4 \%$ and $55.6 \%$, respectively) [38]. A survey in 2007 found that $43 \%$ of the money boys had female sexual partners but only $36 \%$ reported consistent condom use in the last six months [45]. Money boys were more likely to move between major cities to earn extra income from the commercial sex industry and hence they may be a particularly important population for transmitting HIV in different geographical locations [49]. These characteristics may result in the population of money boys becoming the core group for HIV transmission among MSM and the general population $[44,45]$. Further investigations are necessary to understand the behaviours of this sub-population in China.

Moderate to high heterogeneities were observed in the conducted meta-analyses. Given the limited details of study information, we only investigated five potential study characteristics associated with the heterogeneity in the meta-regression model. Recruitment method was found to be the major study characteristic associated with high heterogeneity in the stratified meta-analyses (associated with marital status, percent of MSM who had female sex partners and condom use at last sex act). Furthermore, we postulate that several other factors, including geographical Chinese regions, age of MSM and migrant status, may also be associated with the heterogeneity observed, although these factors could not be assessed by meta-regression due to limited data. Other study variables such as the quality of interviewers, questionnaire or marital status of MSM were not reported in the collated studies but may also contribute to the heterogeneities across studies.

MSM is a population highly susceptible to the emerging HIV epidemic in China [9,50,51]. Further studies on specific sub-populations of MSM, such as money boys, transgender MSM, MSM who inject drugs and bisexual MSM, are necessary to understand their sexual behaviours and contribution to the HIV transmission within and beyond the MSM population. Currently, there are no specific public health interventions targeting bisexual MSM in China. Our results indicate that MSM recruited at MSM venues were more likely to be currently married, had sex with females in last six months, and had unprotected sex with females. Harm-reduction interventions such as distribution of condoms and lubricants to bisexual MSM may be implemented at MSM venues and further expanded to the wider communities. Given the low condom usage between MSM and their regular female partners, public health intervention strategies should particularly target married MSM and their regular female partners. Sexual behaviour and condom use rates with different partnerships should be clearly identified in the future social-behavioural surveys in order to understand the bisexua behaviour among MSM and to curb the spread of HIV transmission from MSM to the overall female population.

\section{Conclusion}

Men who have sex with men are a population that is highly susceptible to the rapid spreading HIV infection in recent years. Our study shows that a substantial proportion of MSM is currently married or has had sex with a female in the past six months. Consistent condom use rates are low among partnerships with regular females. Our findings imply that the general female population, as regular partners of MSM, has a high risk of contracting HIV infection that are bridged from their bisexual male partners. Scale-up of the harm-reduction interventions and health educations for bisexual MSM and their female partners are necessary to halt the further spread of HIV into the general female population.

\section{Acknowledgements}

This study was funded from the following sources: the Australian Government Department of Health and Ageing; the University of New South Wales; the World Bank Global HIV/AIDS Program; and grant no FT0991990 from the Australian Research Council. The views expressed in this publication do not necessarily represent the position of the Australian Government. The Kirby Institute is affiliated with the Faculty of Medicine, University of New South Wales.

\section{Author details}

${ }^{1}$ The Kirby Institute for infection and immunity in society, University of New South Wales, Sydney, Australia. ${ }^{2} \mathrm{CFI}$ Building, Corner of West and Boundary Streets, Darlinghurst, Sydney NSW 2010, Australia. 


\section{Authors' contributions}

All authors were involved in the study design, including setting up the keywords search and the project protocol. EPFC and LZ performed the literature search, quality assessment and data extraction. EPFC performed data analysis. LZ and DPW assisted with data analysis and interpretation. DPW was responsible for the supervision of the project. All authors contributed in writing and editing the manuscript. All authors read and approved the final version of the manuscript.

\section{Competing interests}

The authors declare that they have no competing interests.

Received: 19 April 2011 Accepted: 15 September 2011

Published: 15 September 2011

\section{References}

1. Ministry of Health of the People's Republic of China: China 2010 UNGASS Country Progress Report. Beijing, China: Ministry of Health; 2010.

2. Ministry of Health People's Republic of China, Joint United Nations Programme on HIV/AIDS, World Health Organization: 2009 Estimates for the HIV/AIDS Epidemic in China. Beijing, China; 2010.

3. Chow EPF, Wilson DP, Zhang J, Jing J, Zhang L: Human Immunodeficiency Virus Prevalence Is Increasing Among Men Who Have Sex With Men in China: Findings From a Review and Meta-Analysis. Sexually Transmitted Diseases 2011.

4. Chow EP, Wilson DP, Zhang L: Patterns of condom use among men who have sex with men in China: a systematic review and meta-analysis. AIDS and Behavior 2011.

5. Zhang B, Li X, Chu Q, Wang n, Wang Z, Zhou S, Tong C, Zhang J, Guan W, Cui Z, et al: [A survey of HIV/AIDS related behaviors among 2250 MSM in nine major cities of China]. Chinese Journal of AIDS \& STD 2008, 14(6):541-547.

6. Hu Q, Lu FB, Gong JP, Li YC, Li GB, Liu JH, Chen H, Liao QH: [Knowledge, attitudes, and practice about STD/AIDS among the men who have sex with men in Nanchang City, Jiangxi Province]. Chinese Journal of Health Education 2006, 22(9):647-649.

7. Qu SQ, Zhang DP, Wu YH, Zhu H, Cui Y, Zhao DH, Zhang J: [A Survey of Knowledge, Attitude and Practice Related to HIV/AIDS among Men Who Have Sex with Men in a Northeast City of China]. Chin J STD/AIDS Prev Cont 2002, 8(6):338-340, 370.

8. Yang HT, Ding JP, Chen GH, Li XF, Zhang BC, Guan WH: [Behavioral features of Men Who Have Sex with Men in Jiangsu]. Jiangsu Prev Med 2006, 17(1):1-4.

9. Choi KH, Gibson DR, Han L, Guo Y: High levels of unprotected sex with men and women among men who have sex with men: a potential bridge of HIV transmission in Beijing, China. AIDS Educ Prev 2004, 16(1):19-30.

10. Liu H, Liu Y, Xiao Y, Zhang Q, Shao Y, Zhen L, Li JH, Choi KH: [A survey of the knowledge, attitude, and behaviors on STD/AIDS in men who have sex with men in Beijing]. Chinese Journal of AIDS \& STD 2005, 11(4):268-270

11. Zhang B, Li X, Hu T, Liu D, Shi T: [HIV/AIDS interventions targeting men who have sex with men (MSM): theory and practice]. Chin J STD/AIDS Prev Cont 2000, 6(3):155-157.

12. Wang $\mathrm{CH}$, Yang $Y H$, Lu GJ, Yang XX, Ge LR, Zhao HR, Zhang YQ: [lntervent male male sexual contact AIDS high dangerous behavior and evaluate effection of intervention]. Chinese Journal of Health Laboratory Technology 2007, 17(12):2291-2292.

13. Feng L, Ding X, Lu R, Liu J, Sy A, Ouyang L, Pan C, Yi H, Liu H, Xu J, et al: High HIV prevalence detected in 2006 and 2007 among men who have sex with men in China's largest municipality: an alarming epidemic in Chongqing, China. J Acquir Immune Defic Syndr 2009, 52(1):79-85.

14. He Q, Wang Y, Lin P, Liu Y, Yang F, Fu X, Li Y, Sun B, Li J, Zhao X, et al: Potential bridges for HIV infection to men who have sex with men in Guangzhou, China. AIDS Behav 2006, 10(4 Suppl):S17-23.

15. Guo H, Wu ZY: [Review of study on marital status and sex behavior features of men who have sex with men]. Disease Surveillance 2010, 25(8):651-654

16. Ruan S, Yang H, Zhu Y, Wang M, Ma Y, Zhao J, McFarland W, Raymond HF: Rising HIV prevalence among married and unmarried among men who have sex with men: Jinan, China. AIDS Behav 2009, 13(4):671-676.
17. Guo Y, Li X, Stanton B: HIV-Related Behavioral Studies of Men Who Have Sex with Men in China: A Systematic Review and Recommendations for Future Research. AIDS Behav 2010.

18. Moher D, Liberati A, Tetzlaff J, Altman DG, Grp P: Preferred Reporting Items for Systematic Reviews and Meta-Analyses: The PRISMA Statement. Plos Medicine 2009, 6(7)

19. Boyle MH: Guidelines for evaluating prevalence studies. Evidence-Based Mental Health 1998, 1(2):37-39.

20. Higgins JPT, Thompson SG, Deeks JJ, Altman DG: Measuring inconsistency in meta-analyses. Brit Med J 2003, 327(7414):557-560.

21. Begg CB, Mazumdar M: Operating characteristics of a rank correlation test for publication bias. Biometrics 1994, 50(4):1088-1101.

22. Thompson SG, Sharp SJ: Explaining heterogeneity in meta-analysis: A comparison of methods. Statistics in Medicine 1999, 18(20):2693-2708.

23. Fu LJ, Fang YR, Guo TY: [Investigation of the sexual behaviors among the MSM in Shaoxing City of Zhejiang Province]. Disease Surveillance 2007, 22(12):818-819.

24. Zhang $M$, Wang XD, Yang Y: [Prevalence oh HIV, anti-HCV, syphilis infection and AIDS knowledge among men who have sex with men (MSM) in Urumqi]. Chin J Public Health 2009, 25(9):1075-1076.

25. Chen SC, Luo Y, Cheng J, Ding JM, Dai YZ, Xu K, Chen KK, Chen WY, Shi SF: [Analysis on results of MSM behavioral surveillance on HIV/AIDS]. Disease Surveillance 2007, 22(3):175-177.

26. Cai GF, Ma QQ, Pan XH, Fu L, Xu WX, Shan XR, Yang Q: [HIV/AIDS Related Knowledge, Attitude, Practice and HIV/STD Infection among MSM in Two Cities of Zhejiang Province]. China Preventive Medicine 2008, 9(6):482-485.

27. Liao MZ, Liu XZ, Fu JH, Qian YS, Zhang XF: [Analysis of data of behavioral surveillance in men who have sex with men(MSM) in Shandong Province]. Chinese Journal of AIDS \& STD 2006, 12(6):530-532.

28. Liu $H$, Wang $N$, Zhang $Q$, Shao $Y$, Wu T, Liu $Y$, Jiang HB, Song $Y$, Zhang $L$, Yin WY, et al: [Study of HIV and syphilis infection situation and sexual behavioral characteristics among 416 MSM]. Chinese Journal of AIDS \& STD 2007, 13(3):230-232, 234

29. Wang Y, Zhang HB, Gui ZG, Yang HW, Fan J, Zheng YJ, Liu L: [Analysis of behavior of MSM population and the biological monitoring result in Mianyang city]. Modern Preventive Medicine 2008, 35(19):3780-3783, 3789.

30. Ruan Y, Li D, Li X, Qian HZ, Shi W, Zhang X, Yang Z, Wang C, Liu Y, Yu M, et al: Relationship between syphilis and HIV infections among men who have sex with men in Beijing, China. Sex Transm Dis 2007, 34(8):592-597.

31. Catania JA, Osmond D, Stall RD, Pollack L, Paul JP, Blower S, Binson D, Canchola JA, Mills TC, Fisher $L$, et al: The continuing HIV epidemic among men who have sex with men. American Journal of Public Health 2001, 91(6):907-914.

32. Jeffries WL: Sociodemographic, Sexual, and HIV and Other Sexually Transmitted Disease Risk Profiles of Nonhomosexual-Identified Men Who Have Sex With Men. American Journal of Public Health 2009, 99(6):1042-1045

33. Weinhardt LS, Kelly JA, Brondino MJ, Rotheram-Borus MJ, Kirshenbaum SB, Chesney MA, Remien RH, Morin SF, Lightfoot M, Ehrhardt AA, et al: HIV transmission risk behavior among men and women living with HIV in 4 cities in the United States. Jaids-J Aca Imm Def 2004, 36(5):1057-1066.

34. Prestage G, Hudson J, Bradley J, Down I, Sutherland R, Corrigan N, Gray B, Chalmers B, Batrouney C, Martin P, et al: Three or More Study (TOMS). Sydney: National Centre in HIV Epidemiology and Clinical Research, The University of New South Wales; 2008.

35. Solomon SS, Srikrishnan AK, Sifakis F, Mehta SH, Vasudevan CK, Balakrishnan P, Mayer KH, Solomon S, Celentano DD: The Emerging HIV Epidemic among Men Who have Sex with Men in Tamil Nadu, India: Geographic Diffusion and Bisexual Concurrency. AIDS and Behavior 2010, 14(5):1001-1010

36. Dandona L, Dandona R, Gutierrez JP, Kumar GA, McPherson S, Bertozzi SM, Team AFS: Sex behaviour of men who have sex with men and risk of HIV in Andhra Pradesh, India. AIDS 2005, 19(6):611-619.

37. Zhang B, Li X, Chu Q, Wang N, Wang Z, Zhou S, Tong C, Zhang J, Guan wh, Cui Z, et al: [Correlation between AIDS and homosexuals: A study of 2046 male homosexuals in nine major cities of China]. The Chinese Journal of Human Sexuality 2008, 17(8):6-10.

38. Cai YM, Liu H, Pan P, Hong FC, Zhou H: [Survey of KABP on STD/AIDS among male sexual workers in Shenzhen City]. China Tropical Medicine 2007, 7(11):2131-2133. 
39. Choi KH, Ning Z, Gregorich SE, Pan QC: The influence of social and sexual networks in the spread of HIV and syphilis among men who have sex with men in Shanghai, China. J Acquir Immune Defic Syndr 2007, 45(1):77-84.

40. Li N, Wang Z, Sun GQ, Sun DY: [Analysis of HIV/AIDS sentinel surveillance among high risk population in Henan province in 2006]. Chinese Journal of AIDS \& STD 2007, 13(5):427-429.

41. Xing JM, Zhang KL, Chen X, Zheng J: [Investigation on HIV/AIDS knowledge and sexual behaviors among men who have sex with men in Hunan province]. Chin J Prev Med 2007, 41(6):511-513.

42. Wives of Homosexual Men in China-A Disadvantaged Group. [http:// www.chinadecoded.com/2010/12/02/wives-of-homosexual-men-in-china-adisadvantaged-group/].

43. Varghese B, Maher JE, Peterman TA, Branson BM, Steketee RW: Reducing the risk of sexual HIV transmission-Quantifying the per-act risk for HIV on the basis of choice of partner, sex act, and condom use. Sexually Transmitted Diseases 2002, 29(1):38-43

44. Jing J, Worth H: HIV in China: Understanding the Social Aspects of the Epidemic. Sydney: University of New South Wales Press; 2010.

45. Liu H, Cai Y, Rhodes AG, Hong F: Money boys, HIV risks, and the associations between norms and safer sex: a respondent-driven sampling study in Shenzhen, China. AIDS Behav 2009, 13(4):652-662.

46. Xi SJ, He YF, Zhou XH: [Analysis of influencing factors of risk behaviors associated with AIDS among money boys in Hangzhou, Zhejiang]. Disease Surveillance 2009, 24(11):839-842.

47. He N, Wong FY, Huang ZJ, Ding YY, Fu CW, Smith BD, Young D, Jiang QW: HIV risks among two types of male migrants in Shanghai, China: money boys vs. general male migrants. Aids 2007, 21:S73-S79.

48. Cao NX, Jiang J, Ge FQ: [Study on sexual safety among MSM sex workers]. Chinese Journal of AIDS/STD 2009, 15(6):583-585.

49. Hu LX, Chen YY, Li J, Du J, Zhao BL, Wang LT: [Effect evaluation of health education on prevention of HIV/AIDS among the migrant population]. Chinese Journal of Health Education 2007, 23(6):431-433.

50. Choi KH, Liu H, Guo Y, Han L, Mandel JS, Rutherford GW: Emerging HIV-1 epidemic in China in men who have sex with men. Lancet 2003, 361(9375):2125-2126.

51. Choi KH, Lui H, Guo Y, Han L, Mandel JS: Lack of HIV testing and awareness of HIV infection among men who have sex with men, Beijing, China. AIDS Educ Prev 2006, 18(1):33-43.

52. Wu J, Chen L, Fan HL, Ruan Y: [A survey on the prevalence of HIV-1 and syphilis infection and characteristics of sexual behaviors in MSM (men who have sex with men) living in Shanghai]. J Diagn Concepts Pract 2008, 7(3):296-299.

53. Zhu YW, Ruan SM, Yang H, Wang MH, Zhang CQ, Jia Z, Shi ZL, Zhang MY: [Investigation on AIDS Related Knowledge Risk Behavior and HIV Infection in MSM in Ji'nan City]. Preventive Medicine Tribune 2007, 13(6):490-492.

54. Gu Y, Qu P, Xu L, Luo M, Wang X, Gu J, Zhao L, Lu Y-h, Zhou B: [Survey of knowledge, attitude, behavior and practice related to STI/HIV among male homosexuality in Shenyang]. Chin J Public Health 2004, 20(5):573-574.

55. Liu LY: [Investigation on HIV/AIDS sexual behavior among men who have sex with men in 2006 in city of Mudanjiang]. Med Ani Prev 2008, 24(7):534-535.

56. Wang J, Luo C, Wen YC: [HIV infection and related risk behaviors among MSM in Harbin]. Chinese Journal of AIDS \& STD 2008, 14(1):75.

57. Wang L, Jie Z, Liu F, Zhan Y, Wu YH: [A survey on general conditions and high risk behaviors among men who have sex with men in a city of Heilongjiang province]. Chinese Journal of AIDS \& STD 2006, 12(4):339-340.

58. Zhang D, Bi P, Lv F, Zhang J, Hiller JE: Changes in HIV prevalence and sexual behavior among men who have sex with men in a northern Chinese city: 2002-2006. Journal of Infectection 2007, 55(5):456-463.

59. Zhao H, Wang G, Liu H: [The Investigation Report of Male-to-male Contact During the Voluntary Consulation in Daolin District of Harebin]. Guide of China Medicine 2009, 7(10):181-183.

60. Guo W, Song AJ, Meng HD, Pang L, Rou KM, Wu ZY: [Survey on AIDS/STD risk behaviors and prevalence among men who have sex with men in Langfang, Hebei]. Chinese Journal of Epidemiology 2008, 29(6):545-547.

61. Liu H, Liu Y, Xiao Y: [A Survey on Knowledge, Attitude, Belief and Practice related to HIV/AIDS among MSM]. Chin J STD/AIDS Prev Cont 2001, 7(5):289-291.
62. Ma J, Guo J: [Internet survey of high risk sexual behaviors and sexually transmitted diseases among male homosexuals in Tianjin]. Modern Preventive Medicine 2007, 34(20):3928-3931.

63. Qu L, Yang JY, Zhang XG, Yang YR, Bao ZQ: [Study on HIV and syphilis infections among men who have sex with men in selected cities of Inner Mongolia]. Chin J Epidemiol 2009, 30(10):1089-1090.

64. Zhong F, Lin P, Xu H, Wang Y, Wang M, He Q, Fan L, Li Y, Wen F, Liang Y, et al: Possible Increase in HIV and Syphilis Prevalence Among Men Who Have Sex with Men in Guangzhou, China: Results from a RespondentDriven Sampling Survey. AIDS Behav 2009.

65. Cai YM, Liu H, Pan P, Hong FC, Feng TJ: [Survey of HIV/AIDS related knowledge and high risk behavior among men who have sex with men in Shenzhen using respondent-driven sampling]. South China J Prev Med 2009, 35(1):4-7.

66. He Q, Wang $Y$, Lin $P$, Zhang Z-b, Zhao $X-x, X u$ H-f: [KAP study on AIDS among men who have sex with men in Guangzhou, Guangdong province]. Chinese Journal of Disease Control \& Prevention 2005, 9(2):106-108.

67. Xu YF, Zhou J, Chen SH, Zhou FH, Mo JC, Zhu JJ: [Investigation of HIV Knowledge and High-risk Behavior Among Men Who Have Sex With Men in Nanning]. Journal of Preventive Medicine Information 2008, 24(10):753-755.

68. Miao ZF, Li J, Lei LM, Han X, Zhang XP: [Survey of AIDS-related Knowledge and Behavior in 312 MSM]. Journal of Ningxia Medical University 2009, 31(6):761-762.

69. Feng Y, Wu Z, Detels R, Qin G, Liu L, Wang X, Wang J, Zhang L: HIV/STD prevalence among men who have sex with men in Chengdu, China and associated risk factors for HIV infection. J Acquir Immune Defic Syndr 2010, 53(Suppl 1):S74-80.

70. Lau JT, Wang M, Tse YK, Gu J, Tsui HY, Zhang Y, Wang N, Cheng F: HIVrelated behaviors among men who have sex with men in China: 20052006. AIDS Educ Prev 2009, 21(4):325-339.

71. Xiao Y, Ding X, Li C, Liu J, Sun J, Jia Y: Prevalence and correlates of HIV and syphilis infections among men who have sex with men in Chongqing Municipality, China. Sex Transm Dis 2009, 36(10):647-656.

72. Zhou J, Zhu JJ, Bin H, Zhang L, Yao M, Zhang W, Zhang W, Zhong JY, You QY, Gao L: [A survey of HIV/STD, HBV and HCV infections and risk behaviors among MSM in two central districts of Guiyang city]. Chinese Journal of AIDS \& STD 2008, 14(1):47-48, 51.

73. Xiao Y, Sun J, Li C, Lu F, Allen KL, Vermund SH, Jia Y: Prevalence and correlates of HIV and syphilis infections among men who have sex with men in seven provinces in China with historically low HIV prevalence. J Acquir Immune Defic Syndr 2010, 53(Suppl 1):S66-73.

74. Zhang BC, Zeng Y, Xu H, Li XF, Zhou SJ, Li H, Liao LM, Zhang XM: [Study on 1389 men who have sex with men regarding their HIV high-risk behaviors and associated factors in mainland China in 2004]. Zhonghua Liu Xing Bing Xue Za Zhi 2007, 28(1):32-36.

\section{Pre-publication history}

The pre-publication history for this paper can be accessed here: http://www.biomedcentral.com/1471-2334/11/242/prepub

\section{doi:10.1186/1471-2334-11-242}

Cite this article as: Chow et al:: What is the potential for bisexual men in China to act as a bridge of HIV transmission to the female population? Behavioural evidence from a systematic review and metaanalysis. BMC Infectious Diseases 2011 11:242. 